TRANSACTIONS OF THE

AMERICAN MATHEMATICAL SOCIETY

Volume 362, Number 11, November 2010, Pages 5605-5633

S 0002-9947(2010)04931-1

Article electronically published on June 14, 2010

\title{
A VARIATIONAL PROBLEM ASSOCIATED WITH THE MINIMAL SPEED OF TRAVELLING WAVES FOR SPATIALLY PERIODIC REACTION-DIFFUSION EQUATIONS
}

\author{
XING LIANG, XIAOTAO LIN, AND HIROSHI MATANO
}

\begin{abstract}
We consider the equation $u_{t}=u_{x x}+b(x) u(1-u), x \in \mathbb{R}$, where $b(x)$ is a nonnegative measure on $\mathbb{R}$ that is periodic in $x$. In the case where $b(x)$ is a smooth periodic function, it is known that there exists a travelling wave (more precisely a "pulsating travelling wave") with average speed $c$ if and only if $c \geq c^{*}(b)$, where $c^{*}(b)$ is a certain positive number depending on $b$. This constant $c^{*}(b)$ is called the "minimal speed". In this paper, we first extend this theory by showing the existence of the minimal speed $c^{*}(b)$ for any nonnegative measure $b$ with period $L$. Next we study the question of maximizing $c^{*}(b)$ under the constraint $\int_{[0, L)} b(x) d x=\alpha L$, where $\alpha$ is an arbitrarily given positive constant. This question is closely related to the problem studied by mathematical ecologists in late 1980s but its answer has not been known. We answer this question by proving that the maximum is attained by periodically arrayed Dirac's delta functions $\alpha L \sum_{k \in \mathbb{Z}} \delta(x+k L)$.
\end{abstract}

\section{INTRODUCTION}

Travelling waves describe a wide class of phenomena in combustion physics, chemical kinetics, biology and other natural sciences. From the physical point of view, travelling waves usually describe transition processes. Transition from one equilibrium to another is a typical case, although more complicated situations may arise. Since the classical paper by Kolmogorov, Petrovsky and Piskunov in 1937, travelling waves have been intensively studied. For example, the monograph [13] provides a comprehensive discussion on this subject.

From the ecological point of view, travelling waves typically describe the expansion of the territory of a certain species, including, in particular, the invasion of alien species in a given habitat. Models for biological invasions in spatially periodic environments were first introduced by Shigesada et al. in dimensions 1 and 2 (see [10, 11, 12]). More precisely, they considered spatially segmented habitats where favorable and less favorable (or even unfavorable) zones appear alternately and analyzed how the pattern and scale of spatial fragmentation affect the speed of invasions. In their study, the spatial fragmentation was typically represented by step functions which take two different values periodically. Mathematically, their

Received by the editors January 31, 2008.

2010 Mathematics Subject Classification. Primary 35K57, 35K55, 35P15; Secondary 92D40, 35B10, 35B30, 35B50, 35K15, 28A25.

The first author was partially supported by Japan Society of Promotion of Science and NSFC Grant:10871185.

(C)2010 American Mathematical Society 5605

Reverts to public domain 28 years from publication 
analysis was partly unrigourous as it relied on formal asymptotics of the travelling wave far away from the front.

Berestycki and Hamel [4] and Berestycki, Hamel and Roques [5] extended and mathematically deepened the work of Shigesada et al. significantly, by dealing with much more general equations of the form $u_{t}=\nabla \cdot((A(x) \nabla u))+f(x, u)$ in $\mathbb{R}^{n}$ with rather general smooth periodic coefficients and by developing various mathematical techniques to study the effect of environmental fragmentation rigourously.

Among other things, they proved that, under certain assumptions on the coefficients, there exists $c^{*}>0$ such that the equation has a pulsating travelling wave if and only if $c \geq c^{*}$. Furthermore, they showed that the minimal speed $c^{*}$ is characterized by the following formula:

$$
c^{*}=\min \{c>0 \mid \exists \lambda>0 \text { such that } \mu(c, \lambda)=0\},
$$

where $\mu(c, \lambda)$ is the principal eigenvalue of a certain elliptic operator associated with the linearization of the travelling wave far away from the front. A more detailed account of this result will be stated in Subsection 2.1 in the special context of our problem.

By using a totally different approach, Weinberger [15] also proved the existence of the minimal speed $c^{*}$ of pulsating travelling waves in a more abstract framework. His method relies on the theory of monotone operators and is a generalization of his earlier work [14 to spatially periodic media.

It is important to note that, as far as one-dimensional diffusion equations are concerned, the minimal speed $c^{*}$ coincides with the so-called spreading speed for a large class of monostable nonlinearities. Here the "spreading speed" roughly means the asymptotic speed of an expanding front that starts from a compactly supported initial data (see Definition 2.9 for details). An early study of spreading speeds in multi-dimensional spaces can be found in [2, 3, 14] for the spatially homogeneous case and in [8] for the spatially periodic case. Weinberger [15] then studied the spreading speeds of order-preserving monostable mappings and applied the results to spatially periodic reaction-diffusion equations and lattice systems.

Berestycki, Hamel and Nadirashvili [6] also studied the spreading speed of reaction-diffusion equations in a very general periodically fragmented environment where both the coefficients and the domain itself are periodic. Recently, the same authors [7] studied the spreading speed of reaction-diffusion equations with constant coefficients, but in very general domains which are not necessarily periodic.

In this paper we consider the following equation:

$$
u_{t}=u_{x x}+b(x) u(1-u), x \in \mathbb{R},
$$

where $b(x)$ is either a smooth function or a measure satisfying $b(x) \geq 0$ and $b(x+L) \equiv b(x), x \in \mathbb{R}$, for some $L>0$.

By the above-mentioned work [4, 5, 6, 15, the minimal speed $c^{*}$ of travelling waves is well-defined at least as far as $b(x)$ is a smooth function, and it coincides with the spreading speed. We denote this minimal speed by $c^{*}(b)$. The goal of the present paper is to consider the variational problem

$$
\underset{b}{\operatorname{Maximize}} c^{*}(b)
$$

under the constraint

$$
\int_{[0, L)} b(x) d x=\alpha L
$$


where $\alpha>0$ is an arbitrarily given constant. In other words we want to find out whether or not there exists an optimal $b(x)$ that gives the fastest spreading speed. We will show that the maximum of $c^{*}(b)$ does indeed exist but that it is not attained by any smooth function $b(x)$ but by a measure which is composed of periodically arrayed Dirac delta functions.

In order to study the above problem, we have to consider the case where the coefficient $b(x)$ in (1.1) is a measure. In what follows, we will use the symbol $\bar{b}(x)$ when we need to emphasize the fact that the coefficient is a measure. Thus we will consider the equation

$$
u_{t}=u_{x x}+\bar{b}(x) u(1-u), \quad x \in \mathbb{R},
$$

where $\bar{b}$ is a nonnegative measure satisfying (1.2). We introdcue two important quantities $c^{*}(\bar{b})$ and $c_{e}^{*}(\bar{b})$. The former denotes the minimal speed of travelling waves for equation (1.3). The latter is a quantity associated with a generalized eigenvalue problem (see Definition 2.14 below). It has been known that $c^{*}(b)=c_{e}^{*}(b)$ if $b$ is smooth. As we will see later, $c^{*}(\bar{b})=c_{e}^{*}(\bar{b})$ even if $\bar{b}$ is a measure (Theorem 2.17). We will then show in Theorem 2.18 that the maximum of $c^{*}(\bar{b})$ is attained by $\bar{b}=h$, where

$$
h(x):=\alpha L \sum_{k \in \mathbb{Z}} \delta\left(x-\left(k+\frac{1}{2}\right) L\right),
$$

with $\delta(x)$ being Dirac delta function.

Strictly speaking, we have to distinguish the travelling wave speeds in the positive direction and those in the negative direction (see Definition 2.7). The abovementioned quantities $c^{*}(\bar{b})$ and $c_{e}^{*}(\bar{b})$ are associated with travelling waves in the positive direction. However, as we will see later in Theorem 2.17, the two speeds (positive and negative) are always equal; therefore no ambiguity occurs by not specifying the direction of the travelling wave.

This paper is organized as follows. In Section 2, we introduce basic notation and state the main results. In Section 3, we prove the well-posedness of equation (1.3). In Section 4, we consider a generalized eigenvalue problem associated with equation (1.3). We derive a uniform bound for $c^{*}(b)$ under the constraint (1.2) when $b$ is smooth, and we show the same uniform bound for $c_{e}^{*}(\bar{b})$ when $\bar{b}$ is a measure. In Section 5 , we show that the minimal speed $c^{*}(\bar{b})$ of travelling waves exists when $\bar{b}$ is a measure, and that it coincides with $c_{e}^{*}(\bar{b})$ and also with the spreading speed. Then we complete the proof of Theorems 2.17, 2.18 and 2.19. In Section 6, we prove a lemma on the equicontinuity of solutions along with another technical lemma. These lemmas are used in Sections 3 and 5.

\section{Notation AND MAin RESUlts}

2.1. Basic notation. In this subsection we introduce some notation and recall some known results which will be used later.

In what follows we fix constants $L>0$ and $\alpha>0$. Let $\Lambda(\alpha)$ be the set defined by

$$
\Lambda(\alpha):=\left\{b(x) \in C^{1}(\mathbb{R}) \mid b(x) \geq 0, b(x)=b(x+L) \text { and } \int_{[0, L)} b(x) d x=\alpha L\right\} .
$$


Definition 2.1. $\bar{\Lambda}(\alpha)$ is defined to be the sequential closure of $\Lambda(\alpha)$ in the space of distributions on $\mathbb{R}$. More precisely, $\bar{b} \in \bar{\Lambda}(\alpha)$ if and only if there exists a sequence $\left\{b_{n}\right\}_{n=1}^{\infty}$ in $\Lambda(\alpha)$ such that

$$
\int_{\mathbb{R}} \bar{b}(x) \eta(x) d x=\lim _{n \rightarrow \infty} \int_{\mathbb{R}} b_{n}(x) \eta(x) d x
$$

for any test function $\eta \in C_{0}^{\infty}(\mathbb{R})$, where the left-hand side of (2.1) is a formal integration representing the dual product $\langle\bar{b}, \eta\rangle$.

Since each $b_{n}$ is positive, $\bar{b}$ is a nonnegative distribution. Consequently, $\bar{b}$ is a Radon measure on $\mathbb{R}$. Therefore (2.1) holds for every $\eta \in C_{0}(\mathbb{R})$. We say that $b_{n} \rightarrow \bar{b}$ in the weak* sense if (2.1) holds.

In what follows, we will not distinguish the measure $\bar{b}$ and its density function $\bar{b}(x)$, as long as there is no fear of confusion. Thus we will often use an expression as in the left-hand side of (2.1).

We also note that, since $b_{n}(x)$ is $L$-periodic, $\bar{b}(x)$ is also $L$-periodic in the following sense:

$$
\int_{\mathbb{R}} \bar{b}(x) \eta(x+L) d x=\int_{\mathbb{R}} \bar{b}(x) \eta(x) d x \quad \text { for } \eta \in C_{0}(\mathbb{R}) .
$$

The following lemmas will be useful later:

Lemma 2.2. Let $\left\{b_{n}\right\} \subset \Lambda(\alpha)$ be a sequence converging to some $\bar{b} \in \bar{\Lambda}(\alpha)$ in the weak ${ }^{*}$ sense. Let $\eta(x)$ be a continuous function on $\mathbb{R}$ satisfying

$$
\sum_{k=-\infty}^{\infty} \max _{0 \leq x \leq L}|\eta(x+k L)|<\infty
$$

Then $\eta$ is $\bar{b}$-integrable on $\mathbb{R}$ and the following hold:

$$
\begin{gathered}
\lim _{n \rightarrow \infty} \int_{\mathbb{R}} b_{n}(x) \eta(x) d x=\int_{\mathbb{R}} \bar{b}(x) \eta(x) d x, \\
\int_{\mathbb{R}} \bar{b}(x)|\eta(x)| d x \leq \alpha L \sum_{k=-\infty}^{\infty} \max _{0 \leq x \leq L}|\eta(x+k L)| .
\end{gathered}
$$

Proof. For each integer $M>0$, we define a cutoff function $q_{M}(x)$ by

$$
q_{M}(x)= \begin{cases}0 & \text { for }|x|>(M+1) L, \\ 1 & \text { for }|x| \leq M L, \\ M+1-L^{-1}|x| & \text { otherwise. }\end{cases}
$$

Then, since $q_{M}(x)|\eta(x)|$ is continuous and compactly supported, we have

$$
\int_{\mathbb{R}} \bar{b}(x) q_{M}(x)|\eta(x)| d x=\lim _{n \rightarrow \infty} \int_{\mathbb{R}} b_{n}(x) q_{M}(x)|\eta(x)| d x .
$$

Note also that, since each $b_{n}$ belongs to $\Lambda(\alpha)$,

$$
\begin{aligned}
\int_{\mathbb{R}} b_{n}(x) q_{M}(x)|\eta(x)| d x & =\sum_{k=-\infty}^{\infty} \int_{0}^{L} b_{n}(x) q_{M}(x+k L)|\eta(x+k L)| d x \\
& \leq \alpha L \sum_{k=-\infty}^{\infty} \max _{0 \leq x \leq L}|\eta(x+k L)| .
\end{aligned}
$$


This and (2.6) imply

$$
\int_{\mathbb{R}} \bar{b}(x) q_{M}(x)|\eta(x)| d x \leq \alpha L \sum_{k=-\infty}^{\infty} \max _{0 \leq x \leq L}|\eta(x+k L)|
$$

for $M=1,2,3, \cdots$. Letting $M \rightarrow \infty$ and applying the monotone convergence theorem, we obtain (2.5). Hence $\eta$ is $\bar{b}$-integrable on $\mathbb{R}$.

Next we observe that

$$
\begin{aligned}
\left|\int_{\mathbb{R}} b_{n}(x) \eta(x) d x-\int_{\mathbb{R}} b_{n}(x) q_{M}(x) \eta(x) d x\right| & \leq \int_{\mathbb{R}} b_{n}(x)\left(1-q_{M}(x)\right)|\eta(x)| d x \\
& \leq \alpha L \sum_{|k| \geq M} \max _{0 \leq x \leq L}|\eta(x+k L)| .
\end{aligned}
$$

The assertion (2.4) easily follows from this and (2.6). The lemma is proven.

Corollary 2.3. Let $\left\{b_{n}\right\}, \bar{b}$ and $\eta$ be as in Lemma 2.2 and let $\left\{f_{n}\right\}$ be a sequence of uniformly bounded continuous functions on $\mathbb{R}$ converging to $f$ locally uniformly on $\mathbb{R}$. Then

$$
\int_{\mathbb{R}} \bar{b}(x) f(x) \eta(x) d x=\lim _{n \rightarrow \infty} \int_{\mathbb{R}} b_{n}(x) f_{n}(x) \eta(x) d x .
$$

Proof. Since, by Lemma 2.2

$$
\lim _{n \rightarrow \infty} \int_{\mathbb{R}} b_{n}(x) f(x) \eta(x) d x=\int_{\mathbb{R}} \bar{b}(x) f(x) \eta(x) d x,
$$

it suffices to show that

$$
\lim _{n \rightarrow \infty} \int_{\mathbb{R}} b_{n}(x)\left(f_{n}(x)-f(x)\right) \eta(x) d x=0 .
$$

This follows easily from the estimate

$$
\left|\int_{\mathbb{R}} b_{n}\left(f_{n}-f\right) \eta d x\right| \leq L \alpha \sum_{k=-\infty}^{\infty} \max _{0 \leq x \leq L}|\eta(x+k L)|\left|f_{n}(x+k L)-f(x+k L)\right|
$$

and the fact that $f_{n} \rightarrow f$ locally uniformly on $\mathbb{R}$.

Lemma 2.4. Let $\left\{b_{n}\right\} \subset \Lambda(\alpha)$ be a sequence converging to some $\bar{b} \in \bar{\Lambda}(\alpha)$ in the weak $k^{*}$ sense. Let $\eta(x)$ be an L-periodic continuous function on $\mathbb{R}$. Then

$$
\lim _{n \rightarrow \infty} \int_{[0, L)} b_{n}(x) \eta(x) d x=\int_{[0, L)} \bar{b}(x) \eta(x) d x .
$$

Proof. By the bounded convergence theorem, we have

$$
\int_{[0, L)} \bar{b}(x) \eta(x) d x=\lim _{\varepsilon \rightarrow 0} \int_{\mathbb{R}} \bar{b}(x) p_{\varepsilon}(x) \eta(x) d x,
$$

where $p_{\varepsilon}$ is a cutoff function defined by

$$
p_{\varepsilon}(x)= \begin{cases}0 & \text { for } x \in(-\infty,-\varepsilon) \cup[L, \infty), \\ \varepsilon^{-1}(x+\varepsilon) & \text { for } x \in[-\varepsilon, 0) \\ 1 & \text { for } x \in[0, L-\varepsilon) \\ \varepsilon^{-1}(L-x) & \text { for } x \in[L-\varepsilon, L)\end{cases}
$$

On the other hand, by the $L$-periodicity of $b_{n}$ and $\eta$, we have

$$
\int_{[0, L)} b_{n}(x) \eta(x) d x=\int_{\mathbb{R}} b_{n}(x) p_{\varepsilon}(x) \eta(x) d x \quad(n=1,2,3, \cdots)
$$


for any $0<\varepsilon \leq L / 2$. It follows that

$$
\lim _{n \rightarrow \infty} \int_{[0, L)} b_{n}(x) \eta(x) d x=\lim _{n \rightarrow \infty} \int_{\mathbb{R}} b_{n}(x) p_{\varepsilon}(x) \eta(x) d x=\int_{\mathbb{R}} \bar{b}(x) p_{\varepsilon}(x) \eta(x) d x .
$$

Combining this and (2.8), we obtain the desired identity. The lemma is proven.

We remark that, because of the $L$-periodicity of the measure $\bar{b} \in \bar{\Lambda}(\alpha)$, we can identify $\bar{b}$ with an element $[\bar{b}]$ of the space $C(\mathbb{R} / L \mathbb{Z})^{*}$, namely the dual space of $C(\mathbb{R} / L \mathbb{Z})$, through the relation

$$
\int_{\mathbb{R} / L \mathbb{Z}}[\bar{b}](x)[\eta](x) d x=\int_{[0, L)} \bar{b}(x) \eta(x) d x \quad \text { for any }[\eta] \in C(\mathbb{R} / L \mathbb{Z}),
$$

where $\eta(x)$ is the periodic continuous function on $\mathbb{R}$ associated with $[\eta]$. Thus $\bar{b}$ can be regarded as a Radon measure on $\mathbb{R} / L \mathbb{Z}$. Furthermore, by (2.7), the convergence $b_{n} \rightarrow \bar{b}$ in the weak ${ }^{*}$ sense implies the convergence $\left[b_{n}\right] \rightarrow[\bar{b}]$ in the weak ${ }^{*}$ sense in $C(\mathbb{R} / L \mathbb{Z})^{*}$.

Let us come back to equation (1.3), which we repeat below:

$$
u_{t}=u_{x x}+\bar{b}(x) u(1-u), \quad x \in \mathbb{R} .
$$

We have so far not specified the range of $t$ for this equation, but what we have typically in mind is either $t>0$ or $t \in \mathbb{R}$.

Definition 2.5. Let $I \subset \mathbb{R}$ be any open interval. A continuous function $u(x, t)$ : $\mathbb{R} \times I \rightarrow \mathbb{R}$ is called a weak solution of (1.3) for $t \in I$ (or a solution in the weak sense) if for any $\eta(x, t) \in C_{0}^{\infty}(\mathbb{R} \times I)$,

$$
-\int_{I} \int_{\mathbb{R}} u \eta_{t} d x d t=\int_{I} \int_{\mathbb{R}}\left(u \eta_{x x}+\bar{b}(x) u(1-u) \eta\right) d x d t .
$$

We next consider the following Cauchy problem:

$$
\begin{cases}u_{t}=u_{x x}+\bar{b}(x) u(1-u) & (x \in \mathbb{R}, t>0), \\ u(x, 0)=u_{0}(x) \geq 0 & (x \in \mathbb{R}),\end{cases}
$$

where $u_{0} \in C(\mathbb{R}) \cap L^{\infty}(\mathbb{R})$.

Definition 2.6. A continuous function $u(x, t): \mathbb{R} \times(0, \infty) \rightarrow \mathbb{R}$ is called a mild solution of (2.9) if

$$
\lim _{t \searrow 0} u(x, t)=u_{0}(x) \text { for any } x \in \mathbb{R}
$$

and if it can be written as

$$
\begin{aligned}
u(x, t)= & \int_{\mathbb{R}} G(x-y, t) u_{0}(y) d y \\
& +\int_{0}^{t} \int_{\mathbb{R}} G(x-y, t-s) \bar{b}(y) u(y, s)(1-u(y, s)) d y d s,
\end{aligned}
$$

where

$$
G(x, t):=\frac{1}{\sqrt{4 \pi t}} \exp \left(-\frac{x^{2}}{4 t}\right) .
$$


As we will show in Section 3, a mild solution of (2.9) exists uniquely for any $\bar{b} \in \bar{\Lambda}(\alpha)$ and $u_{0} \in C(\mathbb{R}) \cap L^{\infty}(\mathbb{R})$ with $u_{0} \geq 0$. Furthermore, any mild solution is a weak solution.

It is easily seen that, if $u(x, t)$ is a mild solution of (2.9), then for any constant $\tau \geq 0, u(x, t+\tau)$ is a mild solution of (2.9) with initial data $u(x, \tau)$ (see Remark 3.5.

We call a function $u(x, t)$ on $\mathbb{R} \times \mathbb{R}$ a mild solution of (1.3) for $t \in \mathbb{R}$ if, for any $\tau \in \mathbb{R}, u(x, t+\tau)$ is a mild solution of (2.9) with initial data $u_{0}(x)=u(x, \tau)$.

Definition 2.7. A mild solution $u(x, t)$ of (1.3) for $t \in \mathbb{R}$ is called a travelling wave (in the positive direction) if $0 \leq u(x, t) \leq 1$ and if there exists a constant $T>0$ such that

$$
\begin{array}{cl}
u(x-L, t)=u(x, t+T) & \text { for }(x, t) \in \mathbb{R} \times \mathbb{R}, \\
\lim _{x \rightarrow-\infty} u(x, t)=1, \lim _{x \rightarrow+\infty} u(x, t)=0 & \text { locally uniformly in } t \in \mathbb{R} .
\end{array}
$$

It is called a travelling wave (in the negative direction) if

$$
\begin{array}{cl}
u(x+L, t)=u(x, t+T) & \text { for }(x, t) \in \mathbb{R} \times \mathbb{R}, \\
\lim _{x \rightarrow-\infty} u(x, t)=0, \lim _{x \rightarrow+\infty} u(x, t)=1 & \text { locally uniformly in } t \in \mathbb{R} .
\end{array}
$$

Remark 2.8. In what follows, unless otherwise specified, by a travelling wave we usually mean the one in the positive direction.

Here we call the quantity $c:=L / T$ the speed (or the average speed) of the travelling wave $u(x, t)$.

Berestycki and Hamel [4, Berestycki, Hamel and Roques [5] and Weinberger 15] established the existence of the minimal speed of travelling waves for general monostable nonlinearities $f(x, u)$ satisfying certain conditions, and they also gave an eigenvalue characterization of the minimal speed (see (2.18) below). As one can easily check, the function $f(x, u):=b(x) u(1-u)$ with $b \in \Lambda(\alpha)$ satisfies the assumptions in [4, 5, 15. Therefore, for any $b \in \Lambda(\alpha)$, there exists the minimal travelling wave speed $c^{*}(b)>0$ for equation (1.1) in the following sense:

$$
\begin{cases}c \geq c^{*}(b) \Rightarrow & \text { There exists a travelling wave with speed } c . \\ 0<c<c^{*}(b) \Rightarrow & \text { No travelling wave with speed } c \text { exists. }\end{cases}
$$

To be more precise, $c^{*}(b)$ is defined to be the minimal travelling wave speed in the positive direction. As mentioned in Remark 2.8, one can also define the minimal travelling wave speed in the negative direction, which we denote by $\tilde{c}^{*}(b)$. As we will see in Theorem 2.17, we always have $c^{*}(b)=\tilde{c}^{*}(b)$, even when $b(x)$ is not symmetric; therefore we do not need to distinguish the two minimal wave speeds.

As we have mentioned earlier, $c^{*}(b)$ also coincides with the so-called "spreading speed" of expanding fronts for (1.3), which is defined as follows:

Definition 2.9. A quantity $c^{* *}(\bar{b})>0$ is called the spreading speed (in the positive direction) if for any nonnegative initial data $u_{0} \not \equiv 0$ with compact support, the mild solution $u\left(x, t, u_{0}\right)$ of (1.3) satisfies that

(i) $\lim _{t \rightarrow \infty} u\left(x, t, u_{0}\right)=0$ uniformly in $\{x>c t\}$ if $c>c^{* *}(\bar{b})$,

(ii) $\lim _{t \rightarrow \infty} u\left(x, t, u_{0}\right)=1$ uniformly in $\{0<x<c t\}$ if $0<c<c^{* *}(\bar{b})$. 
A quantity $\tilde{c}^{* *}(\bar{b})>0$ is called the spreading speed (in the negative direction) if for any nonnegative initial data $u_{0} \not \equiv 0$ with compact support, the mild solution $u\left(x, t, u_{0}\right)$ of (1.3) satisfies that

(i) $\lim _{t \rightarrow \infty} u\left(x, t, u_{0}\right)=0$ uniformly in $\{x<-c t\}$ if $c>\tilde{c}^{* *}(\bar{b})$,

(ii) $\lim _{t \rightarrow \infty} u\left(x, t, u_{0}\right)=1$ uniformly in $\{-c t<x<0\}$ if $0<c<\tilde{c}^{* *}(\bar{b})$.

Remark 2.10. Basic properties of the spreading speed in general periodic environments are studied in [4, 5, 6, 8, 15]. It is known, at least for smooth $b$, that the spreading speed $c^{* *}(b)$ coincides with the minimal wave speed $c^{*}(b)$ (and, similarly, $\left.\tilde{c}^{* *}(b)=\tilde{c}^{*}(b)\right)$. As we will show later in Theorem 2.19, we have $c^{* *}(\bar{b})=\tilde{c}^{* *}(\bar{b})$ for any $\bar{b} \in \bar{\Lambda}(\alpha)$.

If $b(x)$ is a smooth periodic function, it is known that any travelling wave $u$ in the positive direction has the following asymptotic expression in the "leading edge", namely the area where $u \approx 0$ :

$$
u(x, t) \sim e^{-\lambda(x-c t)} \psi(x),
$$

where $\psi(x+L) \equiv \psi(x)>0$, and $\lambda>0$ is some constant. Substituting (2.12) into equation (1.1), we obtain the identity

$$
-\psi^{\prime \prime}(x)+2 \lambda \psi^{\prime}(x)-b(x) \psi(x)=\left(\lambda^{2}-\lambda c\right) \psi(x) .
$$

Similarly, for the travelling wave $u$ in the negative direction, we have the following asymptotic expression in the "leading edge":

$$
u(x, t) \sim e^{\lambda(x+c t)} \psi(x),
$$

where $\psi(x+L) \equiv \psi(x)>0$, and $\lambda>0$ is some constant. Hence

$$
-\psi^{\prime \prime}(x)-2 \lambda \psi^{\prime}(x)-b(x) \psi(x)=\left(\lambda^{2}-\lambda c\right) \psi(x) .
$$

These observations motivate us to introduce the following operator, which generalizes the operator on the left-hand side of (2.13) to the case where $b(x)$ is a measure:

Definition 2.11. For $\bar{b} \in \bar{\Lambda}(\alpha), \lambda \in \mathbb{R}$, we define an (unbounded) operator $-L_{\lambda, \bar{b}}$ on the Banach space $\{\psi \in C(\mathbb{R}) \mid \psi(x)=\psi(x+L)\}$ with $\|\psi\|=\max _{x \in \mathbb{R}}|\psi(x)|$ as follows:

$$
-L_{\lambda, \bar{b}} \psi(x)=-\psi^{\prime \prime}(x)+2 \lambda \psi^{\prime}(x)-\bar{b}(x) \psi(x) .
$$

Here the derivatives are understood in the "weak sense" by which we mean that $-L_{\lambda, \bar{b}} \psi=g$ if and only if, for any $\varphi \in C_{0}^{\infty}(\mathbb{R})$,

$$
\int_{\mathbb{R}}\left(-\varphi^{\prime \prime}-2 \lambda \varphi^{\prime}-\bar{b} \varphi\right) \psi d x=\int_{\mathbb{R}} \varphi g d x
$$

Definition 2.12. Given $\bar{b} \in \bar{\Lambda}(\alpha), \lambda \in \mathbb{R}$, we call $\mu(\lambda, \bar{b})$ the principal eigenvalue of the operator $-L_{\lambda, \bar{b}}:=-\psi^{\prime \prime}(x)+2 \lambda \psi^{\prime}(x)-\bar{b}(x) \psi(x)$ if there exists a positive continuous function $\psi$ with $\psi(x) \equiv \psi(x+L)$ satisfying

$$
-\psi^{\prime \prime}(x)+2 \lambda \psi^{\prime}(x)-\bar{b}(x) \psi(x)=\mu(\lambda, \bar{b}) \psi
$$

in the weak sense. Here $\psi$ is called the principal eigenfunction. 
For the principal eigenvalue, the following proposition holds:

Proposition 2.13. For any $\bar{b} \in \bar{\Lambda}(\alpha)$ and $\lambda \in \mathbb{R}$, the principal eigenvalue $\mu(\lambda, \bar{b})$ exists. It is unique and simple and belongs to $\mathbb{R}$.

The above result is well known if $\bar{b}$ is a smooth function. For general $\bar{b} \in$ $\bar{\Lambda}(\alpha)$, we will prove the propositions in Sections 4 and 5 . More precisely we will prove the existence of the principal eigenvalue $\mu(\lambda, \bar{b})$ in Proposition 4.7 by an approximation argument, and we show the uniqueness and simplicity in Proposition 5.12 by converting the problem (2.17) into a more regular eigenvalue problem for a compact positive operator.

We also note that the principal eigenfunction of (2.17) belongs to $H_{l o c}^{1}(\mathbb{R})$, as we will see in Subsection 4.2. Now observe that $\lambda^{2}-\lambda c$ in (2.13) is a constant and that $\psi>0$. Therefore, (2.13) implies that $\mu(\lambda, b)=\lambda^{2}-\lambda c$ if $b$ is smooth. In view of this, we define the following quantities when $\bar{b}$ is a general measure.

Definition 2.14. For $\bar{b} \in \bar{\Lambda}(\alpha)$, we define the minimal speed in the positive direction $c^{*}(\bar{b})$ and a related value $c_{e}^{*}(\bar{b})$ as follows:

$c^{*}(\bar{b}):=\inf \{c>0 \mid$ TW of (1.3) in the positive direction with speed $c$ exists $\}$,

$c_{e}^{*}(\bar{b}):=\inf \left\{c>0 \mid \exists \lambda>0\right.$ such that $\left.\mu(\lambda, \bar{b})=\lambda^{2}-\lambda c\right\}$.

Here "TW" stands for a travelling wave. Similarly, the minimal speeds in the negative directions $\tilde{c}^{*}(\bar{b})$ and $\tilde{c}_{e}^{*}(\bar{b})$ are

$\tilde{c}^{*}(\bar{b}):=\inf \{c>0 \mid$ TW of (1.3) in the negative direction with speed $c$ exists $\}$,

$\tilde{c}_{e}^{*}(\bar{b}):=\inf \left\{c>0 \mid \exists \lambda>0\right.$ such that $\left.\mu(-\lambda, \bar{b})=\lambda^{2}-\lambda c\right\}$.

Here the symbol "inf" is understood in the sense that inf $A=+\infty$ if $A=\emptyset$. If $b(x)$ is a smooth nonnegative function, then by the results of [4, 5, 6] or by those of [15], the following proposition holds.

Proposition 2.15 (4, 5, 6], 15]). For any $b \in \Lambda(\alpha)$, we have

$$
c^{* *}(b)=c^{*}(b)=c_{e}^{*}(b), \tilde{c}^{* *}(b)=\tilde{c}^{*}(b)=\tilde{c}_{e}^{*}(b) .
$$

Note that the relations $c^{* *}(b)=c_{e}^{*}(b), \tilde{c}^{* *}(b)=\tilde{c}_{e}^{*}(b)$ can also be found in the early work of $[8]$. We will see that the conclusion of the above proposition continues to hold, along with the assertion (2.11), even if $b$ is a measure. (See Theorems 2.17 and 2.19)

2.2. Main results. We are now ready to present our main results.

It is shown in [5] that for any $b \in \Lambda(\alpha)$, we have $c^{*}(b) \geq 2 \sqrt{\alpha}=c^{*}(\alpha)$. Our first proposition gives an upper bound on $c^{*}(b)$ :

Proposition 2.16. For any $b \in \Lambda(\alpha)$,

$$
2 \sqrt{\alpha} \leq c^{*}(b) \leq 2 \sqrt{\alpha+\alpha^{2} L^{2}} .
$$

Moreover, if $b \not \equiv \alpha$, then $2 \sqrt{\alpha}<c^{*}(b)$.

As we mentioned above, the inequality $2 \sqrt{\alpha} \leq c^{*}(b)$ is found in [5]. The main novelty of this proposition is the upper bound.

While the lower bound in (2.19) is sharp since the equality holds for $b \equiv \alpha$, it has not been known whether $c^{*}(b)$ attains its maximum in $\Lambda(\alpha)$ or not. The next two theorems show that $c^{*}(b)$ does not attain its maximum in $\Lambda(\alpha)$ but it does in the extended class $\bar{\Lambda}(\alpha)$. 
Theorem 2.17 (Minimal speed). For any $\bar{b} \in \bar{\Lambda}(\alpha)$, it follows that $c^{*}(\bar{b})>0$, and a travelling wave in the positive direction with speed $c$ exists if and only if $c \geq c^{*}(\bar{b})$. Furthermore,

$$
c^{*}(\bar{b})=c_{e}^{*}(\bar{b}) .
$$

Similarly, a travelling wave in the negative direction with speed $c$ exists if and only if $c \geq \tilde{c}^{*}(b)$, and $\tilde{c}^{*}(\bar{b})=\tilde{c}_{e}^{*}(\bar{b})$. Furthermore, for any $\bar{b} \in \bar{\Lambda}(\alpha)$,

$$
c^{*}(\bar{b})=\tilde{c}^{*}(\bar{b}) .
$$

Theorem 2.18 (Optimal coefficient). Let $h$ be as in (1.4). Then

$$
c^{*}(h)=\sup _{b \in \Lambda(\alpha)} c^{*}(b)=\max _{\bar{b} \in \bar{\Lambda}(\alpha)} c^{*}(\bar{b}) .
$$

Furthermore,

$$
c^{*}(h)>c^{*}(b) \quad \text { for any } b \in \Lambda(\alpha) .
$$

Theorem 2.19 (Spreading speed). For any $\bar{b} \in \bar{\Lambda}(\alpha)$, the spreading speed in the positive direction $c^{* *}(\bar{b})$ and that in the negative direction $\tilde{c}^{* *}(\bar{b})$ exist and

$$
c^{* *}(\bar{b})=c^{*}(\bar{b}), \quad \tilde{c}^{* *}(\bar{b})=\tilde{c}^{*}(\bar{b}) .
$$

Consequently,

$$
c^{* *}(\bar{b})=\tilde{c}^{* *}(\bar{b})
$$

To prove Theorem 2.18, the following proposition is important. We will prove it in Subsection 4.3

Proposition 2.20. Let $\left\{b_{n}\right\}$ be a sequence in $\Lambda(\alpha)$ converging to some $\bar{b} \in \bar{\Lambda}(\alpha)$ in the weak $k^{*}$ sense. Then

$$
c_{e}^{*}(\bar{b})=\lim _{n \rightarrow \infty} c_{e}^{*}\left(b_{n}\right)
$$

\section{Reaction-Diffusion equation With a Borel-MEasure COEFFicient}

In this section, we establish the well-posedness of the Cauchy problem (2.9). Throughout this section $\bar{b}$ will denote a given element in $\bar{\Lambda}(\alpha)$.

Theorem 3.1. For any given nonnegative initial data $u_{0} \in C(\mathbb{R}) \cap L^{\infty}(\mathbb{R})$, the problem (2.9) has a unique mild solution. This a mild solution is also a weak solution and it depends continuously on the initial data in the $L^{\infty}$ norm.

Proof. First we show that the solution $u(x, t)$ exists in the weak sense. Let $b_{n} \in$ $\Lambda(\alpha)$ satisfy $b_{n} \rightarrow \bar{b}$ in the weak* sense. Then for any given initial data $u_{0}(x)$, the problem

$$
\left\{\begin{array}{l}
\left(u_{n}\right)_{t}=\left(u_{n}\right)_{x x}+b_{n}(x) u_{n}\left(1-u_{n}\right) \\
u_{n}(x, 0)=u_{0}(x)
\end{array}\right.
$$

has a classical solution $u_{n}(x, t)$ for any $n \in \mathbb{N}$. By the comparison principle, $0 \leq$ $u_{n}(x, t) \leq \max \left\{\left\|u_{0}\right\|_{L^{\infty}(\mathbb{R})}, 1\right\}$. Hence $u_{n}(n \in \mathbb{N})$ are uniformly bounded. Let $0<t_{1}<t_{2}$ be two positive numbers. By Lemma 6.1, which we will prove in Section 6 , the family of solutions $\left\{u_{n}(x, t)\right\}_{n \in \mathbb{N}}$ are uniformly equicontinuous in $(x, t) \in \mathbb{R} \times\left[t_{1}, t_{2}\right]$. Here the modulus of equicontinuity may depend on $t_{1}$ and $t_{2}$. Applying the Arzela-Ascoli theorem, we can choose a subsequence, which we still denote by $\left\{u_{n}(x, t)\right\}$, that converges uniformly in $(x, t) \in[-M, M] \times\left[t_{1}, t_{2}\right]$ 
for every $M>0$ and $0<t_{1}<t_{2}$. The limit function $u(x, t)=\lim _{n \rightarrow \infty} u_{n}(x, t)$ is defined for every $(x, t) \in \mathbb{R} \times(0,+\infty)$ and satisfies

$$
u_{t}=u_{x x}+\bar{b}(x) u(1-u)
$$

in the weak sense. Next we show that $u$ satisfies

$$
\lim _{t \searrow 0} u(x, t)=u_{0}(x) \quad \text { for any } x \in \mathbb{R} .
$$

To see this we first note that, by Lemma 3.2 below, $u$ can be written in the form

$$
\begin{aligned}
u(x, t)= & \int_{\mathbb{R}} G(x-y, t) u_{0}(y) d y \\
& +\int_{0}^{t} \int_{\mathbb{R}} G(x-y, t-s) \bar{b}(y) f(u(y, s)) d y d s,
\end{aligned}
$$

where $f(u):=u(1-u)$. For any $x \in \mathbb{R}$, the first integral satisfies

$$
\int_{\mathbb{R}} G(x-y, t) u_{0}(y) d y \rightarrow u_{0}(x), \text { as } t \rightarrow 0 .
$$

By Lemma 2.2 and (6.1) the second integral can be estimated as follows:

$$
\begin{aligned}
& \left|\int_{0}^{t} \int_{\mathbb{R}} G(x-y, t-s) \bar{b}(y) f(u(y, s)) d y d s\right| \\
\leq & \int_{0}^{t} \alpha L \sum_{k \in \mathbb{Z}}\left|\max _{k L \leq y \leq(k+1) L} G(x-y, t-s) f(u(y, s)) d y\right| d s \\
\leq & D(t+\sqrt{\pi t}),
\end{aligned}
$$

where $D$ is a constant depending on $\|u(\cdot, t)\|_{L^{\infty}(\mathbb{R})}$. Since $\|u(\cdot, t)\|_{L^{\infty}(\mathbb{R})}$ is bounded, we have

$$
\int_{0}^{t} \int_{\mathbb{R}} G(x-y, t-s) \bar{b}(y) f(u(y, s)) d y d s \rightarrow 0 \text { as } t \rightarrow 0 .
$$

Consequently (3.3) holds.

Next, let $u_{0}, \tilde{u}_{0} \in C(\mathbb{R}) \cap L^{\infty}(\mathbb{R})$ be arbitrary and let $u, \tilde{u}$ be the corresponding mild solutions of (2.9), the existence of which has been proven above. Then by Lemma 3.2 below, $w:=u-\tilde{u}$ can be expressed as

$$
\begin{aligned}
w(x, t)=\int_{\mathbb{R}} G( & x-y, t) w_{0}(y) d y \\
& +\int_{0}^{t} \int_{\mathbb{R}} G(x-y, t-s) m(y, s) w(y, s) d y d s,
\end{aligned}
$$

where $m:=\bar{b}(x)(1-u-\tilde{u})$. Define

$$
\rho(t)=\|w(\cdot, t)\|_{L^{\infty}(\mathbb{R})} .
$$

Then, by Lemma 2.2 and (6.1), we have

$$
\rho(t) \leq \rho(0)+M \alpha L \int_{0}^{t} \frac{\rho(s) d s}{\sqrt{4 \pi(t-s)}},
$$


for $t$ in any bounded interval $[0, T]$, where $M$ is a constant depending on $\|1-u-\tilde{u}\|_{L^{\infty}(\mathbb{R})}$ and T. By Lemma 7.7 of Alfaro, Hilhorst, Matano [1], it follows that

$$
\rho(t) \leq e^{M^{2} t / 4}\left(1+\frac{M}{\sqrt{4 \pi}} \int_{0}^{t} \frac{e^{-M^{2} s / 4}}{\sqrt{s}} d s\right) \rho(0)=O\left(e^{M^{2} t / 4}(1+\sqrt{t}) \rho(0)\right) .
$$

Consequently

$$
\|u(\cdot, t)-\tilde{u}(\cdot, t)\|_{L^{\infty}(\mathbb{R})} \leq e^{M^{2} t / 4}\left(1+\frac{M}{\sqrt{4 \pi}} \int_{0}^{t} \frac{e^{-M^{2} s / 4}}{\sqrt{s}} d s\right)\left\|u_{0}-\tilde{u}_{0}\right\|_{L^{\infty}(\mathbb{R})} .
$$

This proves the continuous dependence on the initial data and the uniqueness of the mild solution. The proof of the theorem is complete.

Lemma 3.2. The function $u(x, t)$ in the proof of Theorem 3.1 can be written as

$$
\begin{aligned}
u(x, t)= & \int_{\mathbb{R}} G(x-y, t) u_{0}(y) d y \\
& +\int_{0}^{t} \int_{\mathbb{R}} G(x-y, t-s) \bar{b}(y) u(y, s)(1-u(y, s)) d y d s,
\end{aligned}
$$

where the second integral on the right-hand side is understood as in (2.1).

Proof. We again put $f(u):=u(1-u)$. Since $u_{n}$ is a classical solution of equation (3.1), it is also a mild solution. Hence

$$
u_{n}(x, t)=\int_{\mathbb{R}} G(x-y, t) u_{0}(y)+\int_{0}^{t} \int_{\mathbb{R}} G(x-y, t-s) b_{n}(y) f\left(u_{n}(y, s)\right) d y d s .
$$

To prove this lemma, it is sufficient to prove

$$
\begin{aligned}
\int_{0}^{t} \int_{\mathbb{R}} G(x-y, t-s) \bar{b}(y) f(u(y, s)) d y d s \\
\quad=\lim _{n \rightarrow \infty} \int_{0}^{t} \int_{\mathbb{R}} G(x-y, t-s) b_{n}(y) f\left(u_{n}(y, s)\right) d y d s .
\end{aligned}
$$

Fix $x \in \mathbb{R}$ and $t>0$ arbitrarily and put

$$
\begin{aligned}
& h_{n}(s)=\int_{\mathbb{R}} G(x-y, t-s) b_{n}(y) f\left(u_{n}(y, s)\right) d y, \\
& h(s)=\int_{\mathbb{R}} G(x-y, t-s) \bar{b}(y) f(u(y, s)) d y
\end{aligned}
$$

for $0<s \leq t$. Then, since $f\left(u_{n}(y, s)\right)$ is uniformly bounded and converges to $f(u(y, s))$ locally uniformly in $(y, s) \in \mathbb{R} \times(0, t]$, we see from Corollary 2.3 that

$$
\lim _{n \rightarrow \infty} h_{n}(s)=h(s) \quad(0<s \leq t) .
$$

Furthermore, by (6.1) in Section 6 we have

$$
\left|h_{n}(s)\right| \leq C\left(1+\frac{1}{\sqrt{t-s}}\right) \quad(n=1,2,3, \cdots)
$$

for some constant $C>0$. Consequently, by the Lebesgue convergence theorem,

$$
\lim _{n \rightarrow \infty} \int_{0}^{t} h_{n}(s) d s=\int_{0}^{t} h(s) d s
$$

which proves (3.8). The lemma is proven. 
Theorem 3.1 and its proof show that the mild solution $u$ of (2.9) can always be expressed as the limit of solutions of (3.1). Note that the convergence $u_{n} \rightarrow u$ holds without taking a subsequence of $\left\{u_{n}\right\}$, since the mild solution of (2.9) is unique for each choice of $u_{0}$ and $\bar{b}$.

Using this approximation procedure, we can show that various estimates and properties that hold for (3.1) automatically carry over to (2.9). For example, the following proposition follows directly from Lemma 6.1 in Section 6:

Proposition 3.3. Let $u\left(x, t, u_{0}\right)$ be the mild solution of (2.9) with initial data $u_{0}$. Then for any $t_{0}>0$ and $M>0$, the family of functions

$$
\left\{u\left(x, t_{0}, u_{0}\right) \mid u_{0} \in C(\mathbb{R}),\left\|u_{0}\right\|_{L^{\infty}(\mathbb{R})} \leq M\right\}
$$

is uniformly equicontinuous in $x$.

Similarly, the following proposition is a consequence of the standard comparison principle for (3.1):

Proposition 3.4 (Comparison principle). Let $u\left(x, t, u_{0}\right)$ be as in Proposition 3.3 . Then $u_{0} \leq v_{0}$ implies

$$
u\left(x, t, u_{0}\right) \leq u\left(x, t, v_{0}\right) \quad \text { for } x \in \mathbb{R}, t \geq 0 .
$$

Remark 3.5. If $u(x, t)$ is a mild solution of (2.9), then for any constant $\tau \geq 0$, $u(x, t+\tau)$ is a mild solution of (2.9) with initial data $u(x, \tau)$. This is obvious if $\bar{b}$ is a smooth function, since a mild solution is a classical solution. In the general case, where $\bar{b} \in \bar{\Lambda}(\alpha)$, we can take a sequence of smooth $b_{n}$ with $b_{n} \rightarrow \bar{b}$ (in the weak* sense) and use the approximation argument found in Lemma 3.2

\section{The Linear EIGENVALUe PROBlem}

4.1. Basic estimates. We recall that $\mu(\lambda, b)$ denotes the principal eigenvalue of the problem

$$
-\psi^{\prime \prime}+2 \lambda \psi^{\prime}-b \psi=\mu(\lambda, b) \psi, \quad \psi(x+L) \equiv \psi(x)
$$

(see Definition 2.12). In this subsection, we estimate $\mu(\lambda, b)$ both from above and below. Let $H_{p e r}^{1}$ be the space defined by

$$
H_{\text {per }}^{1}:=\left\{\varphi \in H_{l o c}^{1}(\mathbb{R}) \mid \varphi(x) \equiv \varphi(x+L)\right\}
$$

endowed with the norm

$$
\|\varphi\|_{H_{p e r}^{1}}=\left(\int_{[0, L)}\left(\varphi^{\prime 2}+\varphi^{2}\right) d x\right)^{1 / 2} .
$$

Let

$$
E_{L}:=\left\{\psi \in H_{p e r}^{1} \mid \psi(x)>0, x \in \mathbb{R}\right\} .
$$

Note that the following embedding is compact:

$$
H_{\text {per }}^{1} \hookrightarrow C(\mathbb{R}) \cap L^{\infty}(\mathbb{R}) .
$$

Lemma 4.1. For any $b \in \Lambda(\alpha)$ and any $\psi(x) \in E_{L}$,

$$
\int_{[0, L)} \psi^{\prime 2}(x) d x-\int_{[0, L)} b(x) \psi^{2}(x) d x \geq-\left(\alpha+\alpha^{2} L^{2}\right) \int_{[0, L)} \psi^{2}(x) d x .
$$


Proof. First, for any $x_{1}, x_{2} \in[0, L)$, we have

$$
\psi^{2}\left(x_{2}\right)-\psi^{2}\left(x_{1}\right)=\int_{x_{1}}^{x_{2}} 2 \psi(x) \psi^{\prime}(x) d x .
$$

Hence, for any positive number $k>0$,

$$
\psi^{2}\left(x_{2}\right)-\psi^{2}\left(x_{1}\right) \leq \frac{1}{k} \int_{[0, L)} \psi^{\prime 2}(x) d x+k \int_{[0, L)} \psi^{2}(x) d x .
$$

Multiplying the above inequality by $b\left(x_{2}\right)$ and integrating it by $x_{1} \in[0, L)$ and $x_{2} \in[0, L)$, we get

$$
\begin{aligned}
& L \int_{[0, L)} b\left(x_{2}\right) \psi^{2}\left(x_{2}\right) d x_{2}-\alpha L \int_{[0, L)} \psi^{2}\left(x_{1}\right) d x_{1} \\
& \leq \alpha L^{2}\left(\frac{1}{k} \int_{[0, L)} \psi^{\prime 2}(x) d x+k \int_{[0, L)} \psi^{2}(x) d x\right) .
\end{aligned}
$$

This is equivalent to

$$
\begin{aligned}
L \int_{[0, L)} b(x) & \psi^{2}(x) d x-\alpha L \int_{[0, L)} \psi^{2}(x) d x \\
\leq & \leq \alpha L^{2}\left(\frac{1}{k} \int_{[0, L)} \psi^{\prime 2}(x) d x+k \int_{[0, L)} \psi^{2}(x) d x\right) .
\end{aligned}
$$

Letting $k=\alpha L$, we obtain

$$
\int_{[0, L)} \psi^{\prime 2}(x) d x-\int_{[0, L)} b(x) \psi^{2}(x) d x \geq-\left(\alpha+\alpha^{2} L^{2}\right) \int_{[0, L)} \psi^{2}(x) d x .
$$

Lemma 4.2. For $\lambda \in \mathbb{R}, b \in \Lambda(\alpha)$, it follows that $\mu(\lambda, b) \geq \mu(0, b)$.

Proof. Let $\psi>0$ be the principal eigenfunctions of $-L_{\lambda, b}$. Then

$$
-\psi^{\prime \prime}+2 \lambda \psi^{\prime}-b(x) \psi=\mu(\lambda, b) \psi \text {. }
$$

Multiplying this by $\psi$ and integrating it from 0 to $L$, we have

$$
\int_{[0, L)} \psi^{\prime 2}(x) d x-\int_{[0, L)} b(x) \psi^{2}(x) d x=\mu(\lambda, b) \int_{[0, L)} \psi^{2}(x) d x
$$

hence

$$
\mu(\lambda, b)=\frac{\int_{[0, L)} \psi^{\prime 2}(x) d x-\int_{[0, L)} b(x) \psi^{2}(x) d x}{\int_{[0, L)} \psi^{2}(x) d x} .
$$

On the other hand, for $\mu(0, b)$, we have the variational formula

$$
\mu(0, b)=\min _{\phi \in E_{L}} \frac{\int_{[0, L)} \phi^{\prime 2}(x) d x-\int_{[0, L)} b(x) \phi^{2}(x) d x}{\int_{[0, L)} \phi^{2}(x) d x} .
$$

Consequently $\mu(\lambda, b) \geq \mu(0, b)$.

Lemma 4.3. For any $\lambda \in \mathbb{R}$ and $b \in \Lambda(\alpha)$, it follows that

$$
-\alpha \geq \mu(\lambda, b) \geq-\alpha-\alpha^{2} L^{2} .
$$


Proof. Dividing (2.17) by $\psi(x)$ and integrating it from 0 to $L$, we get

$$
(\mu(\lambda, b)+\alpha) L+\int_{[0, L)}\left(\frac{\psi^{\prime}}{\psi}\right)^{2} d x=0 .
$$

This implies that $\mu(\lambda, b) \leq-\alpha$. By Lemmas 4.1 and 4.2 .

$$
\mu(\lambda, b) \geq \mu(0, b) \geq-\alpha-\alpha^{2} L^{2} .
$$

Lemma 4.4. There exists a constant $F>0$ such that for any $\lambda \in \mathbb{R}, b \in \Lambda(\alpha)$, the principal eigenfunction $\psi \in E_{L}$ of the operator $-L_{\lambda, b}$ satisfies

$$
\frac{\max \psi}{\min \psi} \leq F .
$$

Proof. By equation (4.3), for any $x_{1}, x_{2} \in[0, L)$,

$$
\begin{aligned}
\left|\ln \frac{\psi\left(x_{2}\right)}{\psi\left(x_{1}\right)}\right| & =\left|\ln \psi\left(x_{2}\right)-\ln \psi\left(x_{1}\right)\right|=\left|\int_{x_{1}}^{x_{2}} \frac{\psi^{\prime}}{\psi} d x\right| \\
& \leq \int_{[0, L)}\left|\frac{\psi^{\prime}}{\psi}\right| d x \leq \sqrt{L} \sqrt{\int_{[0, L)}\left(\frac{\psi^{\prime}}{\psi}\right)^{2} d x} \\
& \leq L \sqrt{-(\mu(\lambda, b)+\alpha)} .
\end{aligned}
$$

Then for any $x_{1}, x_{2} \in[0, L]$,

$$
e^{-L \sqrt{-(\mu(\lambda, b)+\alpha})} \leq \frac{\psi\left(x_{1}\right)}{\psi\left(x_{2}\right)} \leq e^{L \sqrt{-(\mu(\lambda, b)+\alpha)}}
$$

This implies

Note that, by (4.2),

$$
\frac{\max \psi}{\min \psi} \leq e^{L \sqrt{-(\mu(\lambda, b)+\alpha)}} .
$$

$$
\sqrt{-(\mu(\lambda, b)+\alpha)} \leq \sqrt{\alpha^{2} L^{2}}=\alpha L .
$$

Therefore, by setting $F=e^{\alpha L^{2}}$, we obtain the desired estimate.

4.2. Uniform bounds of $c^{*}(b)$ for smooth $b$. In this subsection, we will prove Proposition 2.16. First, since the operators $-L_{\lambda, b}$ and $-L_{-\lambda, b}$ are mutually adjoint for any $\lambda \in \mathbb{R}$ and since their principal eigenvalues are real numbers, we have the following formula:

Proposition 4.5. For any $\lambda \in \mathbb{R}$ and $b \in \Lambda(\alpha)$,

$$
\mu(-\lambda, b)=\mu(\lambda, b)
$$

The following proposition easily follows from the definition of $c_{e}^{*}(\bar{b})$ in Definition 2.14 .

Proposition 4.6. For $b \in \Lambda(\alpha)$,

$$
c_{e}^{*}(b)=\min _{\lambda>0} \frac{-\mu(\lambda, b)+\lambda^{2}}{\lambda} .
$$

Proof of Proposition 2.16, By Proposition 2.15, it suffices to show that

$$
2 \sqrt{\alpha} \leq c_{e}^{*}(b) \leq 2 \sqrt{\alpha+\alpha^{2} L^{2}} .
$$

This follows immediately from (4.2) and (4.5). The proposition is proven. 
4.3. Uniform bounds of $c_{e}^{*}(\bar{b})$ when $\bar{b}$ is a measure. In the previous subsection, we discussed the boundedness of $c^{*}(b)$ for $b \in \Lambda(\alpha)$. In this subsection, we derive the same bounds for $c_{e}^{*}(\bar{b})$ when $\bar{b} \in \bar{\Lambda}(\alpha)$. We start with the following proposition:

Proposition 4.7. Let $b_{n}$ be a sequence in $\Lambda(\alpha)$ converging to some $\bar{b}$ in the weak* sense and let $\lambda_{n} \in \mathbb{R}$ be a sequence converging to some $\lambda \in \mathbb{R}$. Then

$$
\mu\left(\lambda_{n}, b_{n}\right) \rightarrow \mu(\lambda, \bar{b}) \quad \text { as } n \rightarrow \infty .
$$

Proof. There exist functions $\psi_{n} \in E_{L}$ with $\left\|\psi_{n}\right\|_{L^{\infty}}=1$ satisfying

$$
-\psi_{n}^{\prime \prime}+2 \lambda_{n} \psi_{n}^{\prime}-\left(\mu\left(\lambda_{n}, b_{n}\right)+b_{n}(x)\right) \psi_{n}=0 .
$$

Multiplying this equation with $\psi_{n}$ and integrating it from 0 to $L$, we get

$$
\int_{[0, L)}\left(\psi_{n}^{\prime}\right)^{2} d x=\int_{[0, L)}\left(\mu\left(\lambda_{n}, b_{n}\right)+b_{n}(x)\right) \psi_{n}^{2} d x .
$$

Hence

$$
\int_{[0, L)}\left(\psi_{n}^{\prime}\right)^{2} d x+\int_{[0, L)}\left(\psi_{n}\right)^{2} d x=\int_{[0, L)}\left(1+\mu\left(\lambda_{n}, b_{n}\right)+b_{n}(x)\right) \psi_{n}^{2} d x .
$$

In view of the fact that $\left\|\psi_{n}\right\|_{L^{\infty}}=1$ and (4.2), we see from the above equation that $\left\{\left\|\psi_{n}\right\|_{H_{p e r}^{1}}\right\}$ is uniformly bounded. Therefore there exists a subsequence $\left\{\psi_{n_{k}}\right\}$ such that

$$
\psi_{n_{k}} \rightarrow \psi \quad \text { weakly in } H_{p e r}^{1} \text { and strongly in } L^{\infty}(\mathbb{R})
$$

and $\mu\left(\lambda_{n_{k}}, b_{n_{k}}\right) \rightarrow \beta$ as $n_{k} \rightarrow \infty$ for some $\psi \in H_{\text {per }}^{1}$ and $\beta \in \mathbb{R}$. Hence

$$
-\psi^{\prime \prime}+2 \lambda \psi^{\prime}-\bar{b} \psi=\beta \psi \quad \text { in the weak sense. }
$$

The strong convergence implies that $\|\psi\|_{L^{\infty}}=1$. Furthermore, since, by Lemma 4.4. there exists a constant $F>0$ such that $\max \psi_{n} / \min \psi_{n} \leq F$, we have $\max \psi / \min \psi \leq F$; hence $\psi>0$. Consequently, $\beta$ is a principal eigenvalue. As we will show in Proposition 5.12, the principal eigenvalue of the operator $-L_{\lambda, \bar{b}}$ is unique. This implies $\mu(\lambda, \bar{b})=\beta$. The proposition is proven.

The following corollary follows immediately from Proposition 4.7 Lemma 4.3 and the fact that every $\bar{b} \in \bar{\Lambda}(\alpha)$ is a weak* limit of the sequence in $\Lambda(\alpha)$ (see (2.1)):

Corollary 4.8. Let $b_{n}$ be a sequence in $\Lambda(\alpha)$ converging to $\bar{b} \in \bar{\Lambda}(\alpha)$ in the weak ${ }^{*}$ sense. Then $\mu\left(\lambda, b_{n}\right) \rightarrow \mu(\lambda, \bar{b})$ locally uniformly in $\lambda \in \mathbb{R}$. In particular,

$$
-\alpha \leq \mu(\lambda, \bar{b}) \leq-\alpha-\alpha^{2} L^{2}
$$

for every $\bar{b} \in \bar{\Lambda}(\alpha)$. 4.7.

We also remark that the continuity of $\lambda \mapsto \mu(\lambda, \bar{b})$ follows easily from Proposition

Proof of Proposition 2.20, By Definition 2.14, we have

$$
\begin{aligned}
& c_{e}^{*}\left(b_{n}\right):=\inf \left\{c>0 \mid \exists \lambda>0 \text { such that } \mu\left(\lambda, b_{n}\right)=\lambda^{2}-\lambda c\right\}, \\
& c_{e}^{*}(\bar{b}):=\inf \left\{c>0 \mid \exists \lambda>0 \text { such that } \mu(\lambda, \bar{b})=\lambda^{2}-\lambda c\right\} .
\end{aligned}
$$

Since $\mu\left(\lambda, b_{n}\right) \rightarrow \mu(\lambda, \bar{b})$ locally uniformly in $\lambda$ and since $\mu\left(\lambda, b_{n}\right)$ is uniformly bounded by Lemma 4.3, we immediately obtain the conclusion.

Combining Propositions 2.16 and 2.20, we obtain the following proposition. 
Proposition 4.9. For any $\bar{b} \in \bar{\Lambda}(\alpha)$,

$$
2 \sqrt{\alpha} \leq c_{e}^{*}(\bar{b}) \leq 2 \sqrt{\alpha+\alpha^{2} L^{2}} .
$$

4.4. Maximizing $c_{e}^{*}(\bar{b})$. In Subsection 4.3 we have shown that $c_{e}^{*}(\bar{b})$ is bounded. In this subsection, we consider the variational problem

$$
\underset{\bar{b} \in \bar{\Lambda}(\alpha)}{\operatorname{Maximize}} c_{e}^{*}(\bar{b})
$$

and show that the maximum is attained by $h(x)$ defined in (1.4).

\section{Lemma 4.10.}

$$
c_{e}^{*}(h)=\max _{\bar{b} \in \bar{\Lambda}(\alpha)} c_{e}^{*}(\bar{b}) .
$$

To prove the above result, we need some auxiliary lemmas.

Lemma 4.11. If there exist $\lambda>0, c>0$ and $\psi \in E_{L}$ such that

$$
-\psi^{\prime \prime}+2 \lambda \psi^{\prime}-\left(\lambda^{2}-\lambda c+h(x)\right) \psi=0,
$$

then $\psi$ is a piecewise $C^{1}$ function and $\psi(L / 2) \geq \psi(x)$ for $x \in \mathbb{R}$.

Proof. First, we just integrate (4.7) from 0 to $L$ to get

$$
\int_{[0, L)}\left(\lambda^{2}-\lambda c+h(x)\right) \psi d x=0 .
$$

This implies $\lambda^{2}-\lambda c<0$.

Since $h(x)=0$ for $x \in(-L / 2, L / 2)$,

$$
-\psi^{\prime \prime}+2 \lambda \psi^{\prime}-\left(\lambda^{2}-\lambda c\right) \psi=0 \quad\left(-\frac{L}{2}<x<\frac{L}{2}\right)
$$

in the classical sense. Furthermore, $\psi$ is continuous up to $x= \pm \frac{L}{2}$ since $E_{L} \subset C(\mathbb{R})$. Consequently, by the classical maximum principle and the positivity of $\psi(x)$, along with the negativity of $\lambda^{2}-\lambda c$, the maximum of $\psi$ must be attained at $x= \pm \frac{L}{2}$. Hence

$$
\psi\left(\frac{L}{2}\right)>\psi(x), \quad \text { for } x \in\left(-\frac{L}{2}, \frac{L}{2}\right) .
$$

From the periodicity of $\psi(x)$, we see that

$$
\psi\left(\frac{L}{2}\right) \geq \psi(x), \quad x \in \mathbb{R} .
$$

Since $\psi$ can be expressed as

$$
\psi(x)=c_{1} e^{\nu_{1} x}+c_{2} e^{\nu_{2} x} \quad \text { in }\left(-\frac{L}{2}, \frac{L}{2}\right)
$$

for some constants $c_{1}, c_{2}, \nu_{1}, \nu_{2}, \psi^{\prime}\left(\frac{L}{2}-0\right)$ and $\psi^{\prime}\left(\frac{L}{2}+0\right)$ both exist. Consequently, $\psi$ is piecewise $C^{1}$ on $\mathbb{R}$.

Lemma 4.12. For any $b \in \Lambda(\alpha), c_{e}^{*}(h)>c_{e}^{*}(b)$.

Proof. In what follows, $c$ will stand for $c_{e}^{*}(h)$ for simplicity. Then, by the definition of $c_{e}^{*}(h)$, there exists $\psi \in E_{L}$ such that

$$
-\psi^{\prime \prime}+2 \lambda \psi^{\prime}-\left(\lambda^{2}-\lambda c+h(x)\right) \psi=0
$$


in the weak sense for some $\lambda>0$. We define the convolution $b * \phi$ for the $L$-periodic function $\phi(x)$ by

$$
b * \phi(x):=\int_{[0, L)} \phi(x-y) b(y) d y .
$$

As is easily seen, this is equivalent to a standard convolution between functions in $C(\mathbb{R} / L \mathbb{Z})$ :

$$
[b] *[\phi](x):=\int_{\mathbb{R} / L \mathbb{Z}}[\phi](x-y)[b](y) d y .
$$

Then one can easily see that $\tilde{\psi}:=b * \psi$ belongs to $E_{L} \cap C^{2}(\mathbb{R})$ since $b$ is $C^{1}$ and $\psi$ is piecewise $C^{1}$. Consequently,

$$
\begin{aligned}
0 & =b *\left(-\psi^{\prime \prime}+2 \lambda \psi^{\prime}-\left(\lambda^{2}-\lambda c+b(x)\right) \psi\right) \\
& =-b * \psi^{\prime \prime}+2 \lambda b * \psi^{\prime}-b *\left(\left(\lambda^{2}-\lambda c+h(x)\right) \psi\right) \\
& =-\tilde{\psi}^{\prime \prime}+2 \lambda \tilde{\psi}^{\prime}-\left(\lambda^{2}-\lambda c+b(x)\right) \tilde{\psi}-(b *(h \psi)-b(b * \psi)) \\
& =-\tilde{\psi}^{\prime \prime}+2 \lambda \tilde{\psi}^{\prime}-\left(\lambda^{2}-\lambda c+b(x)\right) \tilde{\psi}-\left(\alpha L \psi\left(\frac{L}{2}\right) b-b(b * \psi)\right) .
\end{aligned}
$$

Note that

$$
\begin{aligned}
\alpha L \psi\left(\frac{L}{2}\right) b(x)-b(x) b * \psi(x) & =b(x)\left(\alpha L \psi\left(\frac{L}{2}\right)-b * \psi(x)\right) \\
& =b(x)\left(b * \psi\left(\frac{L}{2}\right)-b * \psi(x)\right) \\
& =b(x) b *\left(\psi\left(\frac{L}{2}\right)-\psi(x)\right)(x) \\
& \geq 0, \not \equiv 0
\end{aligned}
$$

by (4.8). It follows that

$$
-\tilde{\psi}^{\prime \prime}+2 \lambda \tilde{\psi}^{\prime}-\left(\lambda^{2}-\lambda c+b(x)\right) \tilde{\psi} \geq 0, \not \equiv 0 .
$$

Now let $\varphi(x)>0$ be the principal eigenfunction for $-L_{-\lambda, b}$. By (4.4),

$$
-\varphi^{\prime \prime}-2 \lambda \varphi^{\prime}-b(x) \varphi=\mu(\lambda, b) \varphi .
$$

Multiplying (4.9) with $\varphi$ and integrating it over $0 \leq x<L$ yield

$$
\begin{aligned}
0 & <\int_{[0, L)} \varphi\left(-\tilde{\psi}^{\prime \prime}+2 \lambda \tilde{\psi}^{\prime}-\left(\lambda^{2}-\lambda c+b\right) \tilde{\psi}\right) d x \\
& =\int_{[0, L)} \tilde{\psi}\left(-\varphi^{\prime \prime}-2 \lambda \varphi^{\prime}-\left(\lambda^{2}-\lambda c+b\right) \varphi\right) d x \\
& =\int_{[0, L)}\left(\mu(\lambda, b)-\left(\lambda^{2}-\lambda c\right)\right) \tilde{\psi} \varphi d x .
\end{aligned}
$$

Consequently, since $\tilde{\psi}, \varphi>0$,

$$
\mu(\lambda, b)>\lambda^{2}-\lambda c .
$$

Combining this and Proposition 4.6, we obtain

$$
c_{e}^{*}(b)<c:=c_{e}^{*}(h) .
$$


Proof of Lemma 4.10. The proof is immediate from Lemma 4.12 and Proposition 2.20 .

\section{Pulsating travelling waves}

In this section we will prove our main results: Theorems 2.17, 2.18 and 2.19] In Subsection 5.1, we prove that the travelling wave in the positive direction with speed $c$ exists if $c \geq c^{*}(\bar{b})$ and $c>0$ and that the travelling wave in the negative direction with speed $c$ exists if $c \geq \tilde{c}^{*}(\bar{b})$ and $c>0$. In Subsection 5.2, we prove $c^{*}(\bar{b})=c_{e}^{*}(\bar{b})$ and $\tilde{c}^{*}(\bar{b})=\tilde{c}_{e}^{*}(\bar{b})$, thereby completing the proof of Theorem 2.17. In Subsection 5.3, we complete the proof of Theorems 2.18 and 2.19. Without loss of generality, in this section, we let $L=1$.

5.1. The minimal wave speed. We apply the general results of Weinberger [15] and Liang and Zhao 9 to show that $c^{*}(\bar{b})$ has the property asserted at the beginning of Theorem 2.17. For this purpose, we need to consider the solution semiflow of (1.3) on the space $C(\mathbb{R} ;[0,1])$ with respect to the local uniform topology, where the convergence $u_{n} \rightarrow u$ means that $u_{n}(x) \rightarrow u(x)$ uniformly on any bounded interval. First, we consider the linear space

$$
B C(\mathbb{R})=\left\{u \mid u \in C(\mathbb{R}) \cap L^{\infty}(\mathbb{R})\right\}
$$

which contains $C(\mathbb{R} ;[0,1])$. Equip $B C(\mathbb{R})$ with the topology of locally uniform convergence. It is easy to show that this linear topological space $B C(\mathbb{R})$ has the following properties.

Proposition 5.1. For each $m \in \mathbb{Z}$ and $n \in \mathbb{N}$, define

$$
\|u\|_{m}^{n}=\sum_{i \in \mathbb{Z}} \frac{\max _{z \in[m+i, m+i+n]}|u(z)|}{2^{|i|}} .
$$

Then each $\|\cdot\|_{m}^{n}$ is a norm on $B C(\mathbb{R})$ and for any $M>0,\|\cdot\|_{m}^{n}$ defines a topology equivalent to the local uniform topology on $C(\mathbb{R} ;[-M, M])$.

Furthermore, for any $u \in B C(\mathbb{R})$,

$$
\|u\|_{m+j}^{1} \leq\|u\|_{m}^{n} \leq \sum_{i=0}^{n-1}\|u\|_{m+i}^{1} \forall m \in \mathbb{Z}, n \in \mathbb{N}, j=0, \ldots, n-1
$$

and

$$
2^{-|m|}\|u\|_{0}^{n} \leq\|u\|_{m}^{n} \leq 2^{|m|}\|u\|_{0}^{n}, \quad \forall m \in \mathbb{Z}, n \in \mathbb{N} .
$$

Proposition 5.2. $C(\mathbb{R} ;[0,1])$ is a bounded closed subset of $B C(\mathbb{R})$. Moreover, $C(\mathbb{R} ;[0,1])$ is complete.

In Section 3, we have shown that the mild solutions of (2.9) depend on the initial data continuously in $L^{\infty}(\mathbb{R})$. The following proposition shows that the continuous dependence also holds with respect to the local uniform topology.

Proposition 5.3. The solutions of (2.9) depend on the initial data continuously in the space $C(\mathbb{R} ;[0,1])$. More precisely, for any $\epsilon>0$ and $T>0$, there is some $\eta>0$ such that for any two solutions $u, v$ of (2.9) with initial data $u_{0}, v_{0} \in C(\mathbb{R} ;[0,1])$, if $\left\|u_{0}-v_{0}\right\|_{0}^{1} \leq \eta$, then $\|u(\cdot, t)-v(\cdot, t)\|_{0}^{1} \leq \epsilon$ for $t \in[0, T]$. 
Proof. Let $w=u-v$ and $w_{0}=u_{0}-v_{0}$. Then

$$
\begin{aligned}
w(x, t)=\int_{\mathbb{R}} G(x-y, t) w_{0}(y) d y & \\
& +\int_{0}^{t} \int_{\mathbb{R}} G(y, t-s) \bar{b}(x-y) m(x-y, s) w(x-y, s) d y d s,
\end{aligned}
$$

where $m(x, s)=1-u(x, s)-v(x, s)$. By the comparison principle and the inequality $0 \leq u_{0}(x), v_{0}(x) \leq 1, \forall x \in \mathbb{R}$, we have $0 \leq u(x, t), v(x, t) \leq 1, \forall x \in \mathbb{R}, t>0$; hence $|m(x, s)| \leq 1$. Let

and

$$
I_{1}(t, x)=\int_{\mathbb{R}} G(x-y, t) w_{0}(y) d y
$$

$$
I_{2}(t, x)=\int_{0}^{t} \int_{\mathbb{R}} G(y, t-s) \bar{b}(x-y) m(x-y, s) w(x-y, s) d y d s .
$$

First, we consider $I_{1}$. For any $\epsilon>0$ and $T>0$ there is some $\eta>0$, such that if $\left\|w_{0}\right\|_{0}^{1}=\left\|I_{1}(0, \cdot)\right\|_{0}^{1}<\eta$, then $\left\|I_{1}(t, \cdot)\right\|_{0}^{1}<\epsilon$ for any $t \in[0, T]$. Next we consider $I_{2}$. By Lemma 2.2.

$$
\begin{aligned}
& \left|\int_{\mathbb{R}} G(y, t-s) \bar{b}(x-y) m(x-y, s) w(x-y, s) d y\right| \\
& \quad \leq \alpha L \sum_{i \in \mathbb{Z}} \max _{y \in[i, i+1]} G(y, t-s) \max _{y \in[i, i+1]}|w(x-y, s)| .
\end{aligned}
$$

Hence we have

$$
\begin{aligned}
\max _{x \in[k, k+1]}\left|I_{2}(t, x)\right| & \leq \alpha L \int_{0}^{t} \max _{x \in[k, k+1]} \sum_{i \in \mathbb{Z}} \max _{y \in[i, i+1]} G(y, t-s) \max _{y \in[i, i+1]}|w(x-y, s)| d s \\
& \leq \alpha L \int_{0}^{t} \sum_{i \in \mathbb{Z}} \max _{y \in[i, i+1]} G(y, t-s) \max _{z \in[k-i-1, k-i+1]}|w(z, s)| d s .
\end{aligned}
$$

This implies that

$$
\begin{aligned}
\left\|I_{2}(t, \cdot)\right\|_{0}^{1} & \leq \alpha L \int_{0}^{t} \sum_{i \in \mathbb{Z}} \max _{y \in[i, i+1]}\left(G(y, t-s) \sum_{k \in \mathbb{Z}} 2^{-|k|} \max _{z \in[k-i-1, k-i+1]}|w(z, s)|\right) d s \\
& =\alpha L \int_{0}^{t} \sum_{i \in \mathbb{Z}} \max _{y \in[i, i+1]} G(y, t-s)\|w(\cdot, s)\|_{-i-1}^{2} d s \\
& \leq \alpha L \int_{0}^{t} \sum_{i \in \mathbb{Z}} \max _{y \in[i, i+1]} G(y, t-s) 2^{|i|+2}\|w(\cdot, s)\|_{0}^{1} .
\end{aligned}
$$

Observe that

$$
\begin{aligned}
\sum_{i \in \mathbb{Z}} \max _{y \in[i, i+1]} G(y, t-s) 2^{|i|+2} & \leq 8 \sum_{i \in \mathbb{Z}} \max _{y \in[i, i+1]} G(y, t-s)\left(2^{y}+2^{-y}\right) \\
& =16 \sum_{i \in \mathbb{Z}} \max _{y \in[i, i+1]} G(y, t-s) 2^{y}=: 16 I .
\end{aligned}
$$

Using the same argument as in (6.1), we have

$$
I \leq \int_{\mathbb{R}} G(y, t-s) 2^{y} d y+2 \max _{y \in \mathbb{R}} G(y, t-s) 2^{y}=e^{(t-s)(\log 2)^{2}}+\frac{e^{(t-s)(\log 2)^{2}}}{\sqrt{\pi(t-s)}} .
$$


Consequently

$$
\left\|I_{2}(t, \cdot)\right\|_{0}^{1} \leq \int_{0}^{t} \frac{C^{\prime}}{\sqrt{t-s}}\|w(\cdot, s)\|_{0}^{1} d s
$$

for some positive constant $C^{\prime}$ depending on $T$. Hence

$$
\|w(\cdot, t)\|_{0}^{1} \leq \epsilon+\int_{0}^{t} \frac{C^{\prime}}{\sqrt{t-s}}\|w(\cdot, s)\|_{0}^{1} d s
$$

provided $\left\|w_{0}\right\|_{0}^{1} \leq \eta$. It follows that

$$
\|w(\cdot, t)\|_{0}^{1} \leq e^{M^{2} t / 4}\left(1+\frac{M}{\sqrt{4 \pi}} \int_{0}^{t} \frac{e^{-M^{2} s / 4}}{\sqrt{s}} d s\right) \epsilon=O\left(e^{M^{2} t / 4}(1+\sqrt{t}) \epsilon\right)
$$

from Lemma 7.7 of [1] (see (3.6) $)$. The proof is complete.

We have shown in Section 3 that for any initial function $u_{0} \in C(\mathbb{R} ;[0,1])$, the mild solution $u\left(x, t, u_{0}\right)$ of (2.9) exists for any $t>0$ and that $u\left(\cdot, t, u_{0}\right) \in C(\mathbb{R} ;[0,1])$. Now define an operator $Q: C(\mathbb{R} ;[0,1]) \times \mathbb{R}^{+} \rightarrow C(\mathbb{R} ;[0,1])$ by

$$
Q_{t}\left(u_{0}\right)(x)=Q\left(u_{0}, t\right)(x)=u\left(x, t, u_{0}\right) .
$$

In the following proposition, we will show that $Q$ is a semiflow on the space $C(\mathbb{R} ;[0,1])$ with respect to the local uniform topology, in other words, with respect to the norm $\|\cdot\|_{0}^{1}$.

Proposition 5.4. $Q$ is a semiflow generated by the mild solution of equation (1.3) in $C(\mathbb{R} ;[0,1])$ (with respect to local uniform topology) in the following sense:

(a) $Q_{0}\left(u_{0}\right)=u_{0}$,

(b) $Q_{t_{1}+t_{2}}=Q_{t_{1}} \circ Q_{t_{2}}$

(c) $Q$ is continuous in $\left(u_{0}, t\right)$,

(d) for each $T>0$, the family of maps $Q_{t}: C(\mathbb{R} ;[0,1]) \rightarrow C(\mathbb{R} ;[0,1]), 0 \leq t \leq$ $T$ is equicontinuous.

Moreover, for any $t>0,\left\{Q_{t}\left(u_{0}\right) \mid u_{0} \in C(\mathbb{R} ;[0,1])\right\}$ is precompact in $C(\mathbb{R} ;[0,1])$.

Proof. Properties (a), (b) are obvious. Property (d) follows from Proposition 5.3. and property (c) from property (d) and the continuity of $u\left(x, t, u_{0}\right)$ in $t$.

The precompactness of $\left\{Q_{t}\left(u_{0}\right) \mid u_{0} \in C(\mathbb{R} ;[0,1])\right\}$ is equivalent to the uniform equicontinuity of the family of functions $\left\{u\left(x, t, u_{0}\right)\right\}_{u_{0} \in C(\mathbb{R} ;[0,1])}$. Therefore it follows from Proposition 3.3 directly. The proof is complete.

In the following lemma, we prove that the semiflow $Q$ is monostable.

Lemma 5.5. $u \equiv 1$ and $u \equiv 0$ are both steady states of (1.3) in the mild sense. Furthermore, for continuous L-periodic initial data $u_{0}$ with $0 \leq u_{0} \leq 1, u_{0} \not \equiv 0$, we have $u\left(x, t, u_{0}\right) \rightarrow 1$ uniformly in $x \in \mathbb{R}$ as $t \rightarrow \infty$.

Proof. Obviously, 1 and 0 are two steady states of (1.3) in the mild sense. Let $v\left(x, t, u_{0}\right)$ be the solution of

$$
v_{t}=v_{x x}
$$

with initial data $v(x, 0)=u_{0}(x)$. From the classical theory of the heat equation, $v\left(x, t, u_{0}\right)>0$ for any $t>0$. 
By Proposition [3.4, we have $0 \leq u\left(x, t, u_{0}\right) \leq 1$. Since $u\left(x, t, u_{0}\right)$ is a mild solution, it can be written as

$$
\begin{aligned}
u\left(x, t, u_{0}\right)= & \int_{\mathbb{R}} G(x-y, t) u_{0}(y) d y \\
& +\int_{0}^{t} \int_{\mathbb{R}} G(x-y, t-s) \bar{b}(y) u\left(y, s, u_{0}\right)\left(1-u\left(y, s, u_{0}\right)\right) d y d s .
\end{aligned}
$$

The second integral is nonnegative; therefore $u\left(x, t, u_{0}\right) \geq v\left(x, t, u_{0}\right)>0$. For any given $t_{1}>0$, we may find a constant $a>0$ such that $u\left(x, t_{1}, u_{0}\right) \geq a$ because $u\left(x, t_{1}, u_{0}\right)$ is periodic with respect to $x$.

By the comparison principle,

$$
u\left(x, t+t_{1}, u_{0}\right) \geq u(x, t, a) \geq v(x, t, a)=a .
$$

Now we choose a sequence $\left\{b_{n}\right\} \subset \Lambda(\alpha)$ converging to $\bar{b}$ in the weak* sense, and we let $u_{n}\left(x, t, u_{0}\right)$ be the solution of (3.1). Then from the proof of Theorem 3.1 and the remark after Lemma 3.2, we see that $u_{n}\left(x, t, u_{0}\right) \rightarrow u\left(x, t, u_{0}\right)$ locally uniformly in $\mathbb{R} \times(0,1]$. Note that $u_{n}(x, t, a)$ is monotone increasing in $t$. In fact

$$
\left(u_{n}\right)_{t}(x, 0, a)=b_{n}(x) a(1-a) \geq 0, \not \equiv 0 .
$$

Hence, by the strong maximum principle applied to $\left(u_{n}\right)_{t}$, we have

$$
\left(u_{n}\right)_{t}(x, t, a)>0 \text { for } t>0 .
$$

It follows that $u(x, t, a)$ is increasing in $t$. Set

$$
P^{+}(x)=\lim _{t \rightarrow \infty} u(x, t, a) .
$$

By the semigroup property of the mild solutions of (1.3), we easily see that $P^{+}(x)$ is a steady state, that is,

$$
u\left(x, t, P^{+}\right)=P^{+}(x) \text { for } x \in \mathbb{R}, t \geq 0 .
$$

We know that $a \leq P^{+} \leq 1$ and that $P^{+}(x)$ is $L$-periodic in $x$ because of the $L$ periodicity of $u(x, t, a)$ for each fixed $t \geq 0$. Here we claim that $P^{+} \equiv 1$. If it is not true, we may find some $x_{0}$ such that

$$
a_{2}=P^{+}\left(x_{0}\right)=\min _{x \in \mathbb{R}} P^{+}(x)<1 .
$$

If $P^{+} \not \equiv a_{2}$, then, $u\left(x, t, P^{+}\right) \geq v\left(x, t, P^{+}\right)>a_{2}$ for any $t>0$. This is a contradiction with (5.3). If $P^{+} \equiv a_{2}$, it is easy to show that $P^{+} \equiv a_{2}$ is not a weak solution of (2.9) with the initial data $a_{2}$. Hence we have $u(x, t, a) \rightarrow 1$ as $t \rightarrow \infty$. This and (5.2) yield $u\left(x, t, u_{0}\right) \rightarrow 1$ as $t \rightarrow \infty$.

Summarizing, for any $t>0, Q_{t}$ has the following properties:

(i) $Q_{t}$ is order-preserving in the sense that if $u_{0}, v_{0} \in C(\mathbb{R} ;[0,1])$ and $u_{0}(x) \leq$ $v_{0}(x)$ on $\mathbb{R}, Q_{t}\left(u_{0}\right)(x) \leq Q_{t}\left(v_{0}\right)(x)$ on $\mathbb{R}$.

(ii) $Q_{t}\left(T_{L}\left(u_{0}\right)\right)=T_{L}\left(Q_{t}\left(u_{0}\right)\right)$, where $T_{L}$ is a shift operator defined by $T_{L}(u)(x)$ $=u(x-L)$.

(iii) $Q_{t}(0)=0$ and $Q_{t}(1)=1$. For any $u_{0} \in C(\mathbb{R} ;[0,1])$ with $u_{0}(x+L) \equiv u_{0}(x)$ and $u \not \equiv 0, Q_{t}\left(u_{0}\right) \rightarrow 1$ in the space $C(\mathbb{R} ;[0,1])$ with respect to the local uniform topology.

(iv) Given $T>0$, the family of maps $Q_{t}: C(\mathbb{R} ;[0,1] \rightarrow C(\mathbb{R} ;[0,1])), 0 \leq t \leq T$, is uniformly equicontinuous with respect to the local uniform topology. 
(v) For each $t>0, Q_{t}(C(\mathbb{R} ;[0,1]))$ is precompact in $C(\mathbb{R} ;[0,1])$ with respect to the local uniform topology.

Thanks to these properties, we can prove the following theorem:

Theorem 5.6. For any $\bar{b} \in \bar{\Lambda}(\alpha)$, the travelling wave in the positive direction with speed $c$ of (1.3) exists for any $c \geq c^{*}(\bar{b}), c>0$, and the travelling wave in the negative direction with speed $c$ exists provided $c \geq \tilde{c}^{*}(\bar{b})$ and $c>0$.

Proof. This theorem can be obtained from the results of Weinberger [15] or a more abstract result of Liang and Zhao [9]. In fact, the above properties (i)-(v) are basically the same as but slightly stronger than Hypotheses 2.1 in [15. Consequently, from Theorem 2.6 of [15] we can get the existence of $c^{*}(\bar{b}), \bar{b} \in \bar{\Lambda}(\alpha)$.

5.2. Proof of $c^{*}(\bar{b})=c_{e}^{*}(\bar{b})$. To prove $c^{*}(\bar{b})=c_{e}^{*}(\bar{b})$, we consider the linearized equation

$$
u_{t}=u_{x x}+\bar{b}(x) u
$$

We define a linear space $\mathbb{X}$ by

$$
\mathbb{X}:=\left\{\phi=e^{\xi_{1} x} \phi_{1}+e^{\xi_{2} x} \phi_{2} \mid \xi_{1}, \xi_{2} \in \mathbb{R}, \phi_{1}, \phi_{2} \in B C(\mathbb{R})\right\}
$$

and the subset

$$
\mathbb{X}_{M}^{\xi}:=\left\{\phi \in \mathbb{X}:|\phi(x)| \leq M e^{\xi|x|}\right\}
$$

for any $M, \xi>0$. Again we equip $\mathbb{X}$ with the local uniform topology. Arguing similarly to the proof of Theorem 3.1 and Proposition 5.3. we can obtain:

Lemma 5.7. For any $\phi \in \mathbb{X}$, the mild solution $u(x, t)$ of the equation

$$
u_{t}=u_{x x}+\bar{b}(x) u
$$

with initial data $u(x, 0)=\phi(x)$ exists for all $t>0$ and is unique. The mild solution is a weak solution and for any $t>0, u(\cdot, t, \phi) \in \mathbb{X}$. Furthermore, the mild solutions depend on the initial data continuously with respect to the local uniform topology on $\mathbb{X}_{M}^{\xi}$ for any $\xi, M>0$.

Define

$$
\Phi_{t}(\phi)(x)=u(x, t, \phi), \quad \forall \phi \in \mathbb{X}
$$

where $u\left(x, t, u_{0}\right)$ is the mild solution of

$$
u_{t}=u_{x x}+\bar{b}(x) u
$$

with initial data $u(x, 0)=u_{0}(x)$. Similarly to the proof of Lemma 6.1 and Proposition 5.4 we can prove the following lemma:

Lemma 5.8. For any $t \geq 0, \Phi_{t}$ is continuous on $\mathbb{X}$ with respect to the local uniform topology.

Next, for any $M>0$, let $A_{M}^{0}=C(\mathbb{R} ;[M, M])$ and $A_{M}^{\xi}=\left\{e^{\xi x} u \mid u \in A_{M}^{0}\right\}$ for $\xi \in \mathbb{R}$. Then we have

Lemma 5.9. $\Phi_{t}\left(A_{M}^{\xi}\right)$ is precompact in $\mathbb{X}$ with respect to the local uniform topology.

In the following lemma, we show that $\Phi_{t}$ is strongly order-preserving.

Lemma 5.10. For any $u_{0} \in B C(\mathbb{R})$ with $u_{0}(x) \geq 0, \forall x \in \mathbb{R}, u_{0} \not \equiv 0$ and any $t>0$, we have $\Phi_{t}\left(u_{0}\right)(x)>0$ for $x \in \mathbb{R}$. 
Proof. Let $u\left(x, t, u_{0}\right)$ be the mild solution of

$$
u_{t}=u_{x x}+\bar{b}(x) u,
$$

with initial data $u_{0}$, and let $v\left(x, t, u_{0}\right)$ be the solution of

$$
v_{t}=v_{x x}
$$

with initial data $u_{0}(x)$. Take a sequence $\left\{b_{n}\right\} \subset \Lambda(\alpha)$ converging to $\bar{b}$ in the weak* sense, and let $u_{n}\left(x, t, u_{0}\right)$ be the solution of

$$
u_{t}=u_{x x}+b_{n}(x) u, u(x, 0)=u_{0}(x) .
$$

Then, arguing as in the proof of Theorem 3.1, we have

$$
u\left(x, t, u_{0}\right)=\lim _{n \rightarrow \infty} u_{n}\left(x, t, u_{0}\right) .
$$

Since $u_{0} \geq 0, b_{n}(x) \geq 0$; we have

$$
u_{n}\left(x, t, u_{0}\right) \geq v\left(x, t, u_{0}\right) .
$$

From the classical theory of the heat equation, $v\left(x, t, u_{0}\right)>0$ for $t>0$. Consequently $\Phi_{t}\left(u_{0}\right)=u\left(x, t, u_{0}\right) \geq v\left(x, t, u_{0}\right)>0$.

Let $\Gamma=\left\{a(x) \in C(\mathbb{R}) \cap L^{\infty}(\mathbb{R}), a(x)=a(x+L)\right\}$. We equip $\Gamma$ with the local uniform topology, which is also equivalent to the $L^{\infty}$ topology on $\Gamma$. For any $\xi \geq 0$, define a linear operator $\mathcal{L}_{t}^{\xi}$ on $\Gamma$ by

$$
\mathcal{L}_{t}^{\xi}(a)=e^{\xi x} \Phi_{t}\left(e^{-\xi x} a\right) .
$$

From the definition and the properties of $\Phi_{t}$, we have

Lemma 5.11. For any $t>0$ and $\xi \geq 0, \mathcal{L}_{t}^{\xi}: \Gamma \rightarrow \Gamma$ is bounded, compact and strongly positive.

Proposition 5.12. If $\psi$ is a principal eigenfunction of $-L_{\lambda, \bar{b}}$, then it is a principal eigenfunction of $\mathcal{L}_{t}^{\lambda}$. Similarly if $\mu(\lambda, \bar{b})$ is a principal eigenvalue of $-L_{\lambda, \bar{b}}$, then $\exp \left(-\mu(\lambda, \bar{b})+\lambda^{2}\right)$ is a principal eigenvalue of $\mathcal{L}_{t}^{\lambda}$. Consequently the principal eigenvalue of $-L_{\lambda, \bar{b}}$ is unique, simple and belongs to $\mathbb{R}$.

Proof. Since $\psi$ is a principal eigenfunction of $-L_{\lambda, \bar{b}}$,

$$
\psi^{\prime \prime}-2 \lambda \psi^{\prime}+\bar{b}(x) \psi=-\mu(\lambda, \bar{b}) \psi \quad \text { in the weak sense. }
$$

Let $\phi=e^{-\lambda x} \psi$. Then the above formula is equivalent to

$$
\phi^{\prime \prime}+\bar{b}(x) \phi=\left(-\mu(\lambda, \bar{b})+\lambda^{2}\right) \phi \quad \text { in the weak sense. }
$$

It is easy to show that $\exp \left(\left(-\mu(\lambda, \bar{b})+\lambda^{2}\right) t\right) \psi$ is the mild solution of $u_{t}=u_{x x}+\bar{b} u$ with $u_{0}(x)=\psi(x)$. Hence,

$$
\Phi_{t}(\phi)=\exp \left(\left(-\mu(\lambda, \bar{b})+\lambda^{2}\right) t\right) \phi .
$$

Finally, we have

$$
\mathcal{L}_{t}^{\lambda}(\psi)=\exp \left(\left(-\mu(\lambda, \bar{b})+\lambda^{2}\right) t\right) \psi .
$$

Since $\mathcal{L}_{t}^{\lambda}$ is a strongly positive compact operator, by the Krein-Rutman theory, its principal eigenvalue is unique and simple. Therefore the principal eigenvalue of $-L_{\lambda, \bar{b}}$, if it exists, is also unique and simple. On the other hand, the existence of a principal eigenvalue of $-L_{\lambda, \bar{b}}$ is shown in Proposition 4.7. The proof of the proposition is complete.

Proposition 2.13 now follows from the above proposition. 
Proposition 5.13. The principal eigenvalue of $\mathcal{L}_{t}^{0}$ is larger than 1 for any $t>0$.

Proof. The principal eigenvalue of $\mathcal{L}_{t}^{0}$ is $e^{-\mu(0, \bar{b}) t}$. Hence, by (4.6), it is larger than 1.

Summarizing, for any $t>0, \Phi_{t}$ has the following properties:

(I) $\Phi_{t}$ is strongly order-preserving in the sense that for any $u_{0} \in B C(\mathbb{R})$ with $u_{0}(x) \geq 0, \forall x \in \mathbb{R}, u \neq 0, \Phi_{t}\left(u_{0}\right)(x)>0$, for $x \in \mathbb{R}$.

(II) $\Phi_{t}\left(T_{L}\left(u_{0}\right)\right)=T_{L}\left(\Phi_{t}\left(u_{0}\right)\right)$, where $T_{L}$ is a shift operator with $T_{L}(u)(x)=$ $u(x-L)$.

(III) For any $t>0$ and $\xi \geq 0$, the operator $\mathcal{L}_{t}^{\xi}: \Gamma \rightarrow \Gamma$ is bounded, compact and strongly positive. Moreover, the principal eigenvalue of $\mathcal{L}_{t}^{0}$ is larger than 1.

In the following lemma, we will show that $Q$ can be dominated by $\Phi$ from above.

Lemma 5.14. Let $\Phi_{t}$ be as defined above and $Q_{t}$ be as in Proposition 5.4. Then for any $u_{0}$ with $u_{0}(x) \geq 0$, we have $Q_{t}\left(u_{0}\right) \leq \Phi_{t}\left(u_{0}\right)$ for any $t>0, x \in \mathbb{R}$.

Proof. We recall that $Q_{t}\left(u_{0}\right)(x)$ is the mild solution $u\left(x, t, u_{0}\right)$ of (2.9) with initial data $u_{0}$ and $\Phi_{t}\left(u_{0}\right)(x)$ is the mild solution $v\left(x, t, u_{0}\right)$ of the equation

$$
v_{t}=v_{x x}+\bar{b}(x) v
$$

with initial data $u_{0}$.

Let $u_{n}\left(x, t, u_{0}\right)$ be the solution of

$$
u_{t}=u_{x x}+b_{n}(x) u(1-u)
$$

with initial data $u_{0}$ and $v_{n}\left(x, t, u_{0}\right)$ be the mild solution of

$$
v_{t}=v_{x x}+b_{n}(x) v
$$

with initial data $u_{0}$ for some $b_{n} \in \Lambda(\alpha)$ such that

$$
u\left(x, t, u_{0}\right)=\lim _{n \rightarrow \infty} u_{n}\left(x, t, u_{0}\right), \quad v\left(x, t, u_{0}\right)=\lim _{n \rightarrow \infty} v_{n}\left(x, t, u_{0}\right) .
$$

From the classical comparison principle for parabolic equations, we have $u_{n}\left(x, t, u_{0}\right)$ $\leq v_{n}\left(x, t, u_{0}\right)$. Hence

$$
Q_{t}\left(u_{0}\right)=u\left(x, t, u_{0}\right) \leq v\left(x, t, u_{0}\right)=\Phi_{t}\left(u_{0}\right)
$$

for any $t>0, x \in \mathbb{R}$.

Modifying the proof of Lemma 5.14 slightly, one can easily get the following lemma, which gives a linear operator that dominates $Q$ from below.

Lemma 5.15. For any $\epsilon$ with $0<\epsilon<1$, define an operator $\Phi_{t}^{\epsilon}$ by

$$
\Phi_{t}^{\epsilon}\left(u_{0}\right)=u^{\epsilon}\left(x, t, u_{0}\right),
$$

where $u^{\epsilon}\left(x, t, u_{0}\right)$ is the mild solution of

$$
u_{t}^{\epsilon}=u_{x x}^{\epsilon}+(1-\epsilon) \bar{b}(x) u^{\epsilon}
$$

with initial data $u_{0} \in B C(\mathbb{R})$. Then $\Phi_{t}^{\epsilon}$ also has the properties (I)-(III). Furthermore, for any given $t_{0}>0, \Phi_{t_{0}}^{\epsilon}\left(u_{0}\right) \leq Q_{t_{0}}\left(u_{0}\right)$ provided $u_{0}(x) \geq 0$ and that $\max \left|u_{0}\right|$ is small enough. 
Proof of Theorem 2.17. We have already shown in Theorem 5.6 the existence of $c^{*}(\bar{b})$. Now, for any $t>0$, we can check that $\Phi_{t}$ satisfies the hypotheses of Theorem 2.5 in [15] and for any $0<\epsilon<1, \Phi_{t}^{\epsilon}$ satisfies the hypotheses of Theorem 2.4 in [15. Moreover, for any $\lambda \geq 0$, the principal eigenvalue of $u_{x x}-2 \lambda u_{x}+(1-\epsilon) \bar{b}(x) u$ under the periodicity conditions converges to the eigenvalue of $u_{x x}-2 \lambda u_{x}+\bar{b}(x) u$ under the same periodicity conditions as $\epsilon \rightarrow 0$. Hence we get

$$
c^{*}(\bar{b})=c_{e}^{*}(\bar{b}), \quad \bar{b} \in \bar{\Lambda}(\alpha) .
$$

Similarly

$$
\tilde{c}^{*}(\bar{b})=\tilde{c}_{e}^{*}(\bar{b}), \quad \bar{b} \in \bar{\Lambda}(\alpha) .
$$

By (4.4) and the definitions of $c_{e}^{*}(b)$ and $\tilde{c}_{e}^{*}(b)$, we have $c_{e}^{*}(b)=\tilde{c}_{e}^{*}(b)$, for smooth $b \in \Lambda(\alpha)$. Thus by Proposition 4.7, we have $c_{e}^{*}(\bar{b})=\tilde{c}_{e}^{*}(\bar{b})$. Combining these, we get $c^{*}(\bar{b})=\tilde{c}^{*}(\bar{b})$.

5.3. Proof of Theorems $\mathbf{2 . 1 8}$ and $\mathbf{2 . 1 9}$. Now we are ready to prove Theorems 2.18 and 2.19 .

Proof of Theorem 2.18, By Lemmas 4.10 and 4.12 ,

$$
c_{e}^{*}(h)=\max _{\bar{b} \in \bar{\Lambda}(\alpha)} c_{e}^{*}(\bar{b})>c_{e}^{*}(b) \text { for any } b \in \Lambda(\alpha) .
$$

On the other hand, in the previous subsection, we have proven that $c_{e}^{*}(\bar{b})=c^{*}(\bar{b})$ for $\bar{b} \in \bar{\Lambda}(\alpha)$. Since $h \in \bar{\Lambda}(\alpha)$, we get

$$
c^{*}(h)=\max _{\bar{b} \in \bar{\Lambda}(\alpha)} c^{*}(\bar{b})>c^{*}(b) \text { for any } b \in \Lambda(\alpha) .
$$

The theorem is proven.

Proof of Theorem [2.19. By the results of Weinberger [15], one can obtain

$$
c^{* *}(\bar{b})=c^{*}(\bar{b}), \quad \tilde{c}^{* *}(\bar{b})=\tilde{c}^{*}(\bar{b}) .
$$

By Theorem 2.17, $c^{*}(\bar{b})=\tilde{c}^{*}(\bar{b})$. Consequently,

$$
c^{* *}(\bar{b})=\tilde{c}^{* *}(\bar{b}) .
$$

The theorem is proven.

\section{Proof of the Lemmas}

In this section we prove the following technical lemma on the equicontinuity of solutions of the Cauchy problem (2.9). This lemma has been used in Sections 3 and 5 .

Lemma 6.1. Let $u\left(x, t, u_{0}, b\right)$ be the solution of (2.9) with $\bar{b}$ replaced by a smooth $b \in \Lambda(\alpha)$ with initial data $u_{0} \in C(\mathbb{R}) \cap L^{\infty}(\mathbb{R})$. Then for any $\epsilon>0, M>0$, $\left\{u\left(x, t, u_{0}, b\right)\right\}_{\left\|u_{0}\right\| \leq M, b \in \Lambda(\alpha)}$ is uniformly equicontinuous in $(x, t) \in \mathbb{R} \times[\epsilon, \infty)$.

Such an equicontinuity result is standard, but what is important about the lemma is that the estimate is uniform in $b \in \Lambda(\alpha)$. For the convenience of the reader, we give an outline of the proof. We begin with the following lemma. 
Lemma 6.2. Let $G(x, t)$ be the heat kernel defined in (2.10). Then there exists a constant $C>0$ such that

$$
\sup _{x \in \mathbb{R}}\left|\int_{\mathbb{R}} G(x-y, \tau) b(y) g(y) d y\right| \leq C \alpha\left(1+\frac{L}{\sqrt{\tau}}\right)\|g\|_{L^{\infty}}
$$

for any $\tau>0, b \in \Lambda(\alpha)$ and $g \in L^{\infty}(\mathbb{R})$.

Proof. Denote by $a(x)$ the integral on the left-hand side of the above inequality. By Lemma 2.2, we have

$$
|a(x)| \leq \alpha L\|g\|_{L^{\infty}} \sum_{k=-\infty}^{\infty} \max _{0 \leq y \leq L} G(x-y-k L, \tau) .
$$

Considering that $G(x, \tau)$ is monotone increasing in $x<0$ and monotone decreasing in $x>0$, we easily find that

$$
\begin{aligned}
\sum_{k=-\infty}^{\infty} \max _{0 \leq y \leq L} G(x-y-k L, \tau) & \leq \frac{1}{L} \int_{\mathbb{R}} G(x-y, \tau) d y+2 \max _{y \in \mathbb{R}} G(y, \tau) \\
& =\frac{1}{L}+\frac{1}{\sqrt{\pi \tau}} .
\end{aligned}
$$

Therefore,

The lemma is proven.

$$
|a(x)| \leq \alpha\|g\|_{L^{\infty}}\left(1+\frac{L}{\sqrt{\pi \tau}}\right) .
$$

Proof of Lemma 6.1. Given $b$ and $u_{0}$, we write $u\left(x, t, u_{0}, b\right)$ as $u(x, t)$ for simplicity, and put $f(u):=u(1-u)$. By the comparison principle, we have

$$
0 \leq u(x, t) \leq \max \left\{1,\left\|u_{0}\right\|_{L^{\infty}}\right\} .
$$

Therefore there exists $K>0$ such that

$$
|f(u(x, t))| \leq K \quad \text { for } x \in \mathbb{R}, t \geq 0 .
$$

In order to prove the lemma, it suffices to show the uniform equicontinuity of $\left\{u\left(x, t+t_{0}, u_{0}, b\right)\right\}_{\left\|u_{0}\right\| \leq M, b \in \Lambda(\alpha), t_{0} \geq 0}$ in $(x, t) \in \mathbb{R} \times[\epsilon, 1]$. Since (6.2) implies

$$
\left\{u\left(x, t+t_{0}, u_{0}, b\right)\right\}_{\left\|u_{0}\right\| \leq M, b \in \Lambda(\alpha)} \subset\left\{u\left(x, t, u_{0}, b\right)\right\}_{\left\|u_{0}\right\| \leq A, b \in \Lambda(\alpha)},
$$

where $A=\max \{1, M\}$, it suffices to prove the uniform equicontinuity of

$$
\left\{u\left(x, t, u_{0}, b\right)\right\}_{\left\|u_{0}\right\| \leq M, b \in \Lambda(\alpha)}
$$

in $(x, t) \in \mathbb{R} \times[\epsilon, 1]$ for any fixed constant $M \geq 1$. We express $u(x, t)$ as

$$
u(x, t)=\int_{\mathbb{R}} G(x-y, t) u_{0}(y) d y+\int_{0}^{t} \int_{\mathbb{R}} G(x-y, s) b(y) f(u(y, t-s)) d y d s .
$$

The uniform equicontinuity of the first term on the right-hand side of (6.4) is easily seen. In what follows we prove the uniform equicontinuity of the second term, which we denote by $\tilde{u}(x, t)$. We choose $t^{*} \in(0, \epsilon]$ and split $\tilde{u}(x, t)$ as $\tilde{u}(x, t)=p(x)+w(x, t)$, where

$$
\begin{gathered}
p(x):=\int_{0}^{t^{*}} \int_{\mathbb{R}} G(x-y, s) b(y) f(u(y, t-s)) d y d s, \\
w(x, t):=\int_{t^{*}}^{t} \int_{\mathbb{R}} G(x-y, s) b(y) f(u(y, t-s)) d y d s .
\end{gathered}
$$


By (6.3) and Lemma 6.2, we have

$$
\sup _{x \in \mathbb{R}}|p(x)| \leq C \alpha K\left(t^{*}+L \sqrt{t^{*}}\right) .
$$

Therefore,

$$
p(x) \rightarrow 0 \quad \text { as } t^{*} \rightarrow 0 \text { uniformly in } \mathbb{R} .
$$

Next we express $w(x, t)$ as

$$
w(x, t)=\int_{0}^{t-t^{*}} \int_{\mathbb{R}} G(x-y, s) q\left(y, t-s-t^{*}\right) d y d s,
$$

where

$$
q(y, s)=\int_{\mathbb{R}} G\left(y-z, t^{*}\right) b(z) f(u(z, s)) d z .
$$

By Lemma 6.2, $q(y, s)$ is uniformly bounded as $u_{0}$ and $b$ vary satisfying $\left\|u_{0}\right\| \leq M$, $b \in \Lambda(\alpha)$. In view of this, we can easily show the uniform equicontinuity of $w(x, t)$ in $(x, t) \in \mathbb{R} \times\left[t^{*}, 1\right]$ either by direct calculations, or by observing that $w$ is a solution of the problem

$$
\left\{\begin{array}{l}
w_{t}=w_{x x}+q\left(x, t-t^{*}\right) \quad\left(x \in \mathbb{R}, t \in\left[t^{*}, 1\right]\right), \\
w\left(x, t^{*}\right)=0
\end{array}\right.
$$

and applying the standard parabolic estimates. Therefore $w(x, t)$ is uniformly equicontinuous in $\mathbb{R} \times[\epsilon, 1]$ for any fixed $t^{*} \in(0, \epsilon]$. Now we let $t^{*} \rightarrow 0$. Then $p(x) \rightarrow 0$ uniformly as shown above; hence $w(x, t) \rightarrow \tilde{u}(x, t)$ uniformly in $\mathbb{R} \times[\epsilon, 1]$. Consequently, $\tilde{u}(x, t)$ is uniform equicontinuous in $\mathbb{R} \times[\epsilon, 1]$; therefore the same is true of $u(x, t)$. The lemma is proven.

\section{ACKNOWLEDGEMENTS}

The authors would like to thank the anonymous referee for his/her helpful comments.

\section{REFERENCES}

1. M. Alfaro, D. Hilhorst, H. Matano, The singular limit of the Allen-Cahn equation and the FitzHugh-Nagumo system, J. Differential Equations 245 (2008), 505-565. MR2428009

2. D. G. Aronson and H. F. Weinberger, Nonlinear diffusion in population genetics, combustion, and nerve pulse propagation, in Partial Differential Equations and Related Topics (J. A. Goldstein, ed.), Lecture Notes in Mathematics, 446, Springer-Verlag, 1975, pp. 5-49. MR 0427837 $(55: 867)$

3. D. G. Aronson and H. F. Weinberger, Multidimensional nonlinear diffusion arising in population dynamics, Adv. Math. 30 (1978), 33-76. MR511740(80a:35013)

4. H. Berestycki, F. Hamel, Front propagation in periodic excitable media, Communications on Pure and Applied Mathematics, LV (2002), 0949-1032. MR1900178 (2003d:35139)

5. H. Berestycki, F. Hamel and L. Roques, Analysis of the periodically fragmented environment model. II. Biological invasions and pulsating traveling fronts, J. Math. Pures Appl. 84 (2005), 1101-1146. MR.2155900 (2006d:35123)

6. H. Berestycki, F. Hamel, N. Nadirashvili, The speed of propagation for KPP type problems. I. Periodic framework, J. Eur. Math. Soc. (JEMS) 7 (2005), 173-213. MR2127993(2005k:35186)

7. H. Berestycki, F. Hamel, N. Nadirashvili, The speed of propagation for KPP type problems. II. General domains, J. Amer. Math. Soc. 23 (2010), 1-34. MR2552247

8. J. Gärtner, M. Freidlin, On the propagation of concentration waves in periodic and random media, Soviet Math. Dokl. 20 (1979), 1282-1286.

9. X. Liang, X. Zhao, Spreading speeds and travelling waves for abstract monotone semiflows and its application, preprint. 
10. N. Kinezaki, K. Kawasaki, F. Takasu, N. Shigesada, Modeling biological invasion into periodically fragmented environments, Theor. Population Biol. 64 (2003), 291-302.

11. N. Shigesada, K. Kawasaki, Biological Invasions: Theory and Practice, Oxford Series in Ecology and Evolution, Oxford Univ. Press, Oxford, 1997.

12. N. Shigesada, K. Kawasaki, E. Teramoto, Traveling periodic waves in heterogeneous environments, Theor. Population Biol. 30 (1986), 143-160. MR850456 (87h:92086)

13. Aizik I. Volpert, Vitaly A. Volpert and Vladimir A. Volpert, Traveling Wave Solutions of Parabolic Systems, Translations of Math. Monographs 140, Amer. Math. Soc. 1994. MR1297766 (96c:35092)

14. H. F. Weinberger, Long-time behavior of a class of biological models, SIAM J. Math. Anal., 13 (1982), 353-396. MR653463 (83f:35019)

15. H. F. Weinberger, On spreading speeds and traveling waves for growth and migration models in a periodic habitat, J. Math. Biol. 45 (2002), 511-548. MR.1943224 (2004b:92043a)

Department of Mathematics, University of Science and Technology of China, China

E-mail address: xliang@ustc.edu.cn

Graduate School of Mathematical Sciences, University of Tokyo, Japan

Graduate School of Mathematical Sciences, University of Tokyo, Japan

E-mail address: matano@ms.u-tokyo.ac.jp 